\title{
IDENTIFICATION OF SHALLOW SEA MODELS
}

\author{
P. G. J. TEN BRUMMELHUIS \\ Department of Applied Mathematics, University of Twente, P.O. Box 217, 7500 AE Enschede, The Netherlands* \\ A. W. HEEMINK \\ Department of Applied Mathematics and Computer Sciences, Delft University of Technology, P.O. Box 356, 2628 AJ Delft, \\ The Netherlands \\ AND \\ H. F. P. VAN DEN BOOGAARD \\ Delft Hydraulics, P.O. Box 177, 2600 MH Delft, The Netherlands
}

\begin{abstract}
SUMMARY
In this paper we consider a parameter estimation procedure for shallow sea models. The method is formulated as a minimization problem. An adjoint model is used to calculate the gradient of the criterion which is to be minimized. In order to obtain a robust estimation method, the uncertainty of the open boundary conditions can be taken into account by allowing random noise inputs to act on the open boundaries. This method avoids the possibility that boundary errors are interpreted by the estimation procedure as parameter fluctuations. We apply the parameter estimation method to identify a shallow sea model of the entire European continental shelf. First, a space-varying bottom friction coefficient is estimated simultaneously with the depth. The second application is the estimation of the parameterization of the wind stress coefficient as a function of the wind velocity. Finally, an uncertain open boundary condition is included. It is shown that in this case the parameter estimation procedure does become more robust and produces more realistic estimates. Furthermore, an estimate of the open boundary conditions is also obtained.
\end{abstract}

KEY WORDS Tidal models Maximum likelihood Modelling uncertain boundaries Parameter estimation

\section{INTRODUCTION}

Shallow sea models are often used to describe the tidal movement in coastal waters and estuaries. This tidal movement is enforced by a tidal wave that enters the area through one of its open boundaries. The propagation of the wave is governed by the shallow water equations, while the tidal force is represented by the open boundary conditions. Before a numerical shallow sea model can be used, it has to be established that it is reliable. The model has to be adjusted so that the model outcome represents a series of observed data as closely as possible. The adjustment of the model implies that some quantities appearing in the model must be specified. These may be the bottom friction coefficients, the parameters of the open boundary conditions or the geometry of the problem. This procedure is called the calibration of the model and is usually done by means of trial and error, which is a very time consuming process. Another major difficulty is the lack of

* Present address: Delft Hydraulics, P.O. Box 152, 8300 AD Emmeloord, the Netherlands 
insight into the complex behaviour of the model. As a consequence only a very few uncertain parameters can be determined by hand. In this paper we introduce a parameter estimation technique to perform the calibration automatically.

If the model is one dimensional and the governing equations are the de St. Venant equations, it is possible to use a Kalman filter (see Reference 1)

(a) to estimate parameters while taking into account the uncertainties that are associated with the deterministic model and the open boundary conditions so that model errors, or more importantly, boundary errors, do not result in unrealistic parameter estimates,

(b) to process the data up to the present time in order to correct the results of the model and determine the optimal initial conditions for a prediction model.

However, if the model is two dimensional, computational problems arise and a direct application of Kalman filtering techniques is out of the question. Therefore, if we focus our attention on two-dimensional shallow sea models, alternative algorithms have to be developed to accomplish the above-mentioned goals. In Reference 2, a first, heuristic approach was developed and applied using simulated data. In this paper we improve this method, provide a sound mathematical basis, and use it for a number of real-life applications.

Section 2 describes a method to estimate uncertain parameters in large-scale models. The method is very robust in the sense that it also produces reliable results in the presence of uncertainties in the open boundaries. Allowing random noise to act on the open boundary conditions leads to a stochastic parameter estimation problem. By defining an error criterion that measures the difference between the model outcome and the observed data, the estimation problem is treated as a minimization problem. The estimation procedure is based on the Maximum Likelihood (ML) method. The original idea of using the ML method for the estimation of uncertain parameters in stochastic models goes back to the 1960s (see References 3-5). However, it leads to a Two-Point Boundary Value Problem (TPBVP) that can usually only be solved at relatively high computational cost. Therefore, we make use of the hyperbolic nature of the model equations to obtain an efficient algorithm.

The parameter estimation is performed with a gradient-based algorithm to minimize the error criterion. The solution of the TPBVP determines the gradient of the error criterion completely without any further calculations. This is the result of applying the method of Chavent in a stochastic environment. This method (see Reference 6) is well known in the field of deterministic parameter estimation theory to derive the exact gradient of a criterion. If it assumed that except for the uncertain parameters, the model and the boundary conditions are perfect, the parameter estimation problem becomes a deterministic one. In this case our approach reduces to the approach introduced by Chavent.

In Section 3, the parameter estimation procedure is employed to identify a number of uncertain parameters of a shallow water flow model of the entire European continental shelf. We first discuss a number of deterministic parameter estimation problems where we assume that, except for the uncertain parameters, the model and the (open) boundary conditions are perfect. As a consequence, the parameter estimation procedure interprets the differences between the model results and the data completely in terms of adaptations of the parameters. This procedure is first applied to the simultaneous estimation of space-varying bottom friction coefficients and a spacevarying depth. Second, the parameterization of the wind stress as function of the wind velocity is identified. Finally, we discuss a stochastic parameter estimation problem where the uncertainty of the open boundary conditions is taken into account to prevent boundary errors causing unrealistic parameter estimates. In this case the bottom friction coefficients, the depth and the time-varying open boundary conditions are estimated simultaneously. As a result, the estimates of the bottom friction coefficient and the depth become more realistic than in the case where the 
open boundary conditions were assumed to be perfect. Moreover, a correction of the open boundary conditions is also obtained. This can be used to gain insight into the accuracy of these conditions.

\section{PARAMETER ESTIMATION THEORY}

\subsection{Shallow sea models}

The dynamic equations of shallow sea models are the so-called shalkow water equations, stating the conservation of mass and momentum (see Reference 7):

$$
\begin{array}{r}
\frac{\partial u}{\partial t}+u \frac{\partial u}{\partial x}+v \frac{\partial u}{\partial y}-2 \omega v+\frac{g u \sqrt{ }\left(u^{2}+v^{2}\right)}{C^{2}(D+h)}+g \frac{\partial h}{\partial x}+\frac{1}{\rho_{\mathrm{w}}} \frac{\partial p_{\mathrm{a}}}{\partial x}-\frac{\rho_{\mathrm{a}} C_{\mathrm{d}} V_{x} \sqrt{ }\left(V_{x}^{2}+V_{y}^{2}\right)}{\rho_{\mathrm{w}}(D+h)}=0 \\
\frac{\partial v}{\partial t}+u \frac{\partial v}{\partial x}+v \frac{\partial v}{\partial y}+2 \omega u+\frac{g v \sqrt{ }\left(u^{2}+v^{2}\right)}{C^{2}(D+h)}+g \frac{\partial h}{\partial y}+\frac{1}{\rho_{\mathrm{w}}} \frac{\partial p_{\mathrm{a}}}{\partial y}-\frac{\rho_{\mathrm{a}} C_{\mathrm{d}} V_{x} \sqrt{ }\left(V_{x}^{2}+V_{y}^{2}\right)}{\rho_{\mathrm{w}}(D+h)}=0 \\
\frac{\partial h}{\partial t}+\frac{\partial}{\partial x}[(D+h) u]+\frac{\partial}{\partial y}[(D+y) v]=0
\end{array}
$$

where

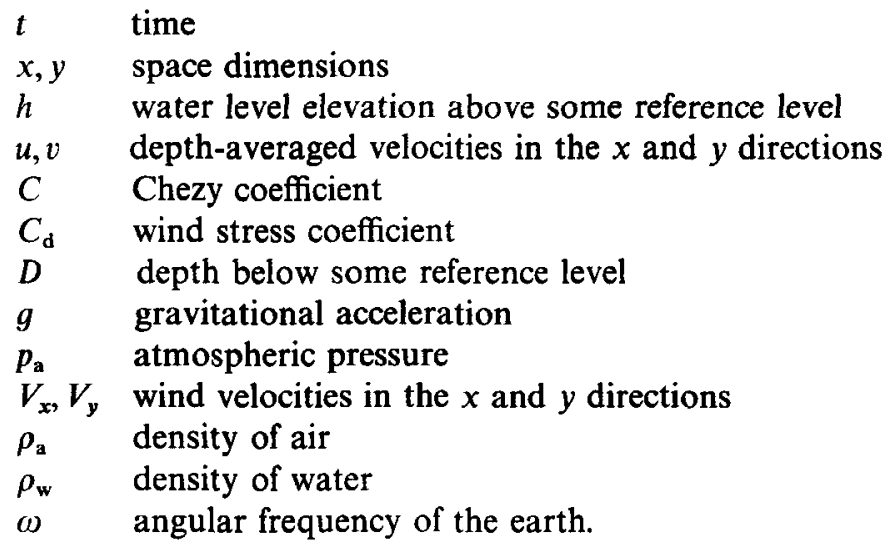

At closed boundaries the perpendicular velocity component is set equal to zero, and at open boundaries the water level is given as a known function of time. However, due to the non-linearity of the dynamics, an additional condition has to be imposed at open boundaries in case of inflow. This condition, stating that the parallel velocity component vanishes at these boundaries, is necessary to ensure the well posedness of the problem (see Reference 8).

In order to approximate the partial differential equations (1)-(3), we define a space-staggered grid (see Figure 1) and employ an ADI finite difference scheme. ${ }^{8}$ In describing the finite difference method, we consider a simpler form of the non-linear shallow water equations by neglecting the advection and the wind stress terms and linearizing the bottom friction terms.

$$
\begin{gathered}
\frac{\partial h}{\partial t}+D\left(\frac{\partial u}{\partial x}+\frac{\partial v}{\partial y}\right)=0, \\
\frac{\partial u}{\partial t}+g \frac{\partial h}{\partial x}+f v+\lambda \frac{u}{D}=0, \\
\frac{\partial v}{\partial t}+g \frac{\partial h}{\partial y}-f u+\lambda \frac{v}{D}=0 .
\end{gathered}
$$




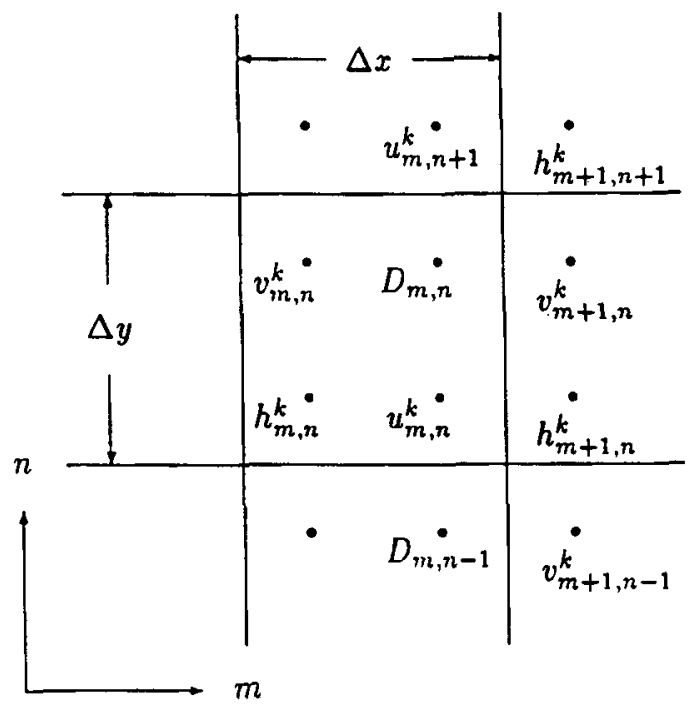

Figure 1. The computational grid

The reasons for this simplification are threefold.

(1) The large-scale application that we discuss in this paper deals with the tidal motion on the entire European continental shelf where the effect of advection is relatively small. This does not mean that these terms are neglected in the numerical representation, but that we concentrate our analysis on the other terms.

(2) The adequate numerical representation of the advection terms involves quite complex formulae ${ }^{8}$ which do not provide any insight into the global characteristics of the numerical model.

(3) The discretization of non-linear bottom friction and wind stress terms is relatively straightforward.

The characteristic of an ADI scheme is the splitting of the time step from $k$ to $k+1$ in two half-time steps. Suppose that the state is defined as $\underline{X}_{k}=\left(\ldots, u_{m, n}^{k}, v_{m, n}^{k}, h_{m, n}^{k}, \ldots\right)^{\mathrm{T}}$, then in the first part of the computation the velocity $u_{m, n}^{k+1 / 2}$ is determined explicitly, based on $u_{m, n}^{k}, v_{m, n}^{k}$ and $h_{m, n}^{k}$, while the remaining variables $u_{m, n}^{k+1 / 2}$ and $h_{m, n}^{k+1 / 2}$ are found as the solution of the implicit equations:

$$
\begin{gathered}
\frac{2}{\Delta t}\left(h_{m, n}^{k+1 / 2}-h_{m, n}^{k}\right)+\frac{D}{\Delta x}\left(u_{m, n}^{k+1 / 2}-u_{m-1, n}^{k+1 / 2}\right)+\frac{D}{\Delta y}\left(v_{m, n}^{k}-v_{m, n-1}^{k}\right)=0, \\
\frac{2}{\Delta t}\left(v_{m, n}^{k+1 / 2}-v_{m, n}^{k}\right)+\frac{g}{\Delta y}\left(h_{m, n+1}^{k}-h_{m, n}^{k}\right)+\frac{f}{4}\left(u_{m-1, n}^{k}+u_{m-1, n+1}^{k}+u_{m, n}^{k}+u_{m, n+1}^{k}\right)+\frac{\lambda_{m, n}}{D} v_{m, n}^{k+1 / 2}=0, \\
\frac{2}{\Delta t}\left(u_{m, n}^{k+1 / 2}-u_{m, n}^{k}\right)+\frac{g}{\Delta x}\left(h_{m+1, n}^{k+1 / 2}-h_{m, n}^{k+1 / 2}\right)-\frac{f}{4}\left(v_{m, n-1}^{k+1 / 2}+v_{m, n}^{k+1 / 2}+v_{m+1, n}^{k+1 / 2}+v_{m+1, n-1}^{k+1 / 2}\right)+\frac{\lambda_{m, n}}{D} u_{m, n}^{k+1 / 2}=0 .
\end{gathered}
$$

In the second part, the roles of the $m$ and $n$ directions are changed; the velocity component $u_{m, n}^{k+1}$ is based on $u_{m, n}^{k+1 / 2}, v_{m, n}^{k+1 / 2}$ and $h_{m, n}^{k+1 / 2}$, and $v_{m, n}^{k+1}$ and $h_{m, n}^{k+1}$ are again the solutions of the 
implicit equations:

$$
\begin{gathered}
\frac{2}{\Delta t}\left(v_{m, n}^{k+1}-v_{m, n}^{k+1 / 2}\right)+\frac{g}{\Delta y}\left(h_{m, n+1}^{k+1}-h_{m, n}^{k+1}\right)+\frac{f}{4}\left(u_{m-1, n}^{k+1}+u_{m-1, n+1}^{k+1}+u_{m, n}^{k+1}+u_{m, n+1}^{k+1}\right)+\frac{\lambda_{m, n}}{D} v_{m, n}^{k+1}=0 \\
\frac{2}{\Delta t}\left(h_{m, n}^{k+1}-h_{m, n}^{k+1 / 2}\right)+\frac{D}{\Delta x}\left(u_{m, n}^{k+1 / 2}-u_{m-1, n}^{k+1 / 2}\right)+\frac{D}{\Delta y}\left(v_{m, n}^{k+1}-v_{m, n}^{k+1}\right)=0 \\
\frac{2}{\Delta t}\left(u_{m, n}^{k+1}-u_{m, n}^{k+1 / 2}\right)+\frac{g}{\Delta x}\left(h_{m+1, n}^{k+1 / 2}-h_{m, n}^{k+1 / 2}\right)-\frac{f}{4}\left(v_{m, n}^{k+1 / 2}+v_{m, n}^{k+1 / 2}+v_{m+1, n}^{k+1 / 2}+v_{m+1, n-1}^{k+1 / 2}\right)+\frac{\lambda_{m, n}}{D} u_{m, n}^{k+1}=0
\end{gathered}
$$

To complete the numerical model, Dirichlet conditions are imposed at the boundaries. They ensure the well posedness of the tidal motion prcblem.

The primary aim of this article is to develop a calibration method for general shallow sea models rather than developing these models themselves. So it is obvious that explicit reference to the detailed numerical representations will only be necessary when dealing with some special issues. Therefore, the numerical model is formally written as the discrete system,

$$
\underline{X}_{k+1}=\underline{f}\left(\underline{p}, \underline{X}_{k}\right)+B \underline{u}_{k+1},
$$

where $\underline{X}_{k}$ is defined as the state of the system. This contains the water level and velocity components in all the grid points. Furthermore, $\underline{u}_{k}$ represents the boundary forcing at time $k$. We include in our notation the fact that the function $f$ is non-linear with respect to $\underline{X}_{k}$. The vector $\underline{p}$ contains the uncertain parameters in the model, e.g. space-varying bottom friction or wind stress coefficients.

Usually, an open boundary is represented by means of a series of harmonic constituents

$$
h_{m_{\mathrm{b}}, n_{\mathrm{b}}}^{k}=\sum_{i=1}^{N}\left(A_{i}\right)_{\left(m_{\mathrm{b}}, n_{\mathrm{b}}\right)} \cos \left[\omega_{i} k \Delta t-\left(\phi_{i}\right)_{\left(m_{\mathrm{b}}, n_{\mathrm{b}}\right)}\right]
$$

where

$$
\begin{array}{ll}
\left(m_{\mathrm{b}}, n_{\mathrm{b}}\right) & \text { co-ordinates along the open boundaries } \\
\left(A_{i}\right)_{\left(m_{\mathrm{b}}, n_{\mathrm{b}}\right)} & \text { the amplitude of the } i \text { th component at }\left(m_{\mathrm{b}}, n_{\mathrm{b}}\right) \\
\omega_{i} & \text { the angular frequency of the ith component } \\
\left(\phi_{i}\right)_{\left(m_{\mathrm{b}}, n_{\mathrm{b}}\right)} \text { the phase of the } i \text { th component at }\left(m_{\mathrm{b}}, n_{\mathrm{b}}\right) .
\end{array}
$$

Due to measurement errors that corrupt the harmonic analysis and the limited number of tidal constituents that can be taken into account, an unknown part can be added to the right-hand side of equation (14) to represent the uncertainties:

$$
h_{\left(m_{\mathrm{b}}, n_{\mathrm{b}}\right)}^{k}=\sum_{i=1}^{N}\left(A_{i}\right)_{\left(m_{\mathrm{b}}, n_{\mathrm{b}}\right)} \cos \left[\omega_{i} k \Delta t-\left(\phi_{i}\right)_{\left(m_{\mathrm{b}}, n_{\mathrm{b}}\right)}\right]+\text { 'unknown part'. }
$$

Since we are dealing with tidal motion problems, this 'unknown part' can also be described by a harmonic sequence

$$
\text { 'unknown part' }=\sum_{j=1}^{j_{\max }}\left(\hat{A}_{j}\right)_{\left(m_{\mathrm{b}}, n_{\mathrm{b}}\right)} \cos \left[\omega_{j} k \Delta t-\left(\hat{\phi}_{j}\right)_{\left(m_{\mathrm{b}}, n_{\mathrm{b}}\right)}\right]
$$


The amplitudes $\left(\hat{A}_{j}\right)_{\left(m_{\mathrm{b}}, n_{\mathrm{b}}\right)}$ and the phases $\left(\hat{\phi}_{j}\right)_{\left(m_{\mathrm{b}}, n_{\mathrm{b}}\right)}$ can now be conceived as unknown but time-invariant model parameters. They can be estimated simultaneously with the other parameters. Because it can be assumed that the spatial variations of the amplitudes $\hat{A}_{j}$ and the phases $\hat{\phi}_{j}$ are relatively small, we can estimate amplitudes and phases in a limited number of open boundary points $\left(m_{\mathrm{b}}^{*}, n_{\mathrm{b}}^{*}\right)$. For the open boundary points in between $\left(\hat{A}_{j}\right)_{\left(m_{\mathrm{b}}, m_{\mathrm{b}}\right)}$ and $\left(\hat{\phi}_{j}\right)_{\left(m_{\mathrm{b}}, m_{\mathrm{b}}\right)}$, these are found by interpolation.

A fundamentally different approach is that where the uncertainty in the open boundary conditions is treated stochastically. Instead of $\left(\hat{A}_{j}\right)_{(m \xi, n \mathrm{~b})}$ and $\left(\hat{\phi}_{j}\right)_{(m \mathrm{k}, n \mathrm{k})}$, we introduce stochastic processes $\left\{q_{m b, n}^{k} \mid k=1,2, \ldots, N\right\}$ at the open boundary points with co-ordinates $\left(m_{\mathrm{b}}^{*}, n_{\mathrm{b}}^{*}\right)$. Similar to the assumption that the 'unknown part' satisfies equation (16), the time evolution of $q_{m \xi, n \mathrm{~b}}^{k}$ must be given. This can be accomplished by assuming that $q_{m b, n \mathrm{~b}}^{k}$ evolves in time according to an AR(1)-model which is driven by a noise process $\eta_{m b, n \mathbf{b}}^{k+1}$ with known statistics

$$
q_{m b, n \mathrm{~b}}^{k+1}=\rho q_{m b, n b}^{k}+\eta_{m b, n b}^{k+1} \text {. }
$$

The fact that the time dependence does not have to be strictly specified is a great advantage in this stochastic approach. The stochastic noisy forcing term $\eta_{m b, n}^{k+1}$ in equation (17) makes the entire tidal model stochastic. If the noise sequence $\eta^{k}$ at the point $\left(m_{\mathrm{b}}^{*}, n_{\mathrm{b}}^{*}\right)$ is denoted by $\eta_{i}^{k}$, the system noise $\underline{w}_{k}=\left(\eta_{1}^{k}, \eta_{2}^{k}, \ldots, \eta_{i_{\max }^{k}}^{k}\right)^{\mathrm{T}}$ and a new state vector $\underline{Y}_{k}$ is defined as $\underline{Y}_{k}=\left(\underline{X}_{k}, q_{i}^{k}\right)^{\mathbf{T}}=\left(\underline{X}_{k}, q_{1}^{k}, q_{2}^{k}, \ldots, q_{i_{\max }}^{k}\right)^{\mathrm{T}}$. This stochastic model can now be formally written as

$$
\underline{Y}_{k+1}=\underline{f}\left(\underline{p}, \underline{Y}_{k}\right)+B \underline{u}_{k+1}+G \underline{w}_{k+1},
$$

where $G$ is the random input matrix and $\underline{w}_{k}$ the Gaussian system noise with zero mean and covariance matrix $Q$.

Observations $\underline{z}_{k}$ are assumed to be available according to

$$
\underline{z}_{k}=H \underline{Y}_{k}+\underline{v}_{k},
$$

where $H$ is the measurement matrix and $\underline{v}_{k}$ the Gaussian measurement noise with zero mean and covariance matrix $R$.

This prescription is the basic model formulation used throughout this paper.

\subsection{The maximum likelihood approach}

The method introduced in this paper tries to find a trajectory, the solution of equations (18) and (19), that best fits the observed data series. This trajectory minimizes a measure (the error criterion) of the difference between the model outcome and the observed data, taking into account the covariances of the measurement and system noise processes. Moreover, this minimizing trajectory is parametrized by the unknown parameters in the dynamic equations and the uncertain open boundary conditions. In our model we assume that the values of the parameters are constant in time, whereas the errors in the open boundary prescription have a time-dependent nature: the 'unknown part' of this boundary is a function of time. Despite the different character of these two error sources, the estimations of the parameters and the open boundary are performed simultaneously, by a method that is based on the ML method.

The simultaneous estimation of parameters and states is performed by maximizing a likelihood function $L\left(\underline{p}, \underline{Y}_{k}\right)$. Usually this is treated as the minimization of $\mathscr{T}\left(\underline{p}, \underline{Y}_{k}\right)$ with $\mathscr{T}\left(\underline{p}, \underline{Y}_{k}\right)=$ $-\log L\left(\underline{p}, \underline{Y}_{k}\right)$ and $\mathscr{T}\left(\underline{p}, \underline{Y}_{k}\right)$ is refered to as the error criterion. The criterion considered here consists of two terms, one representing the measurement error and the other the system error. So the minimization of $\mathscr{T}$ with respect to states and parameters can also be interpreted as finding realizations of the measurement and system noise sequences that have minimum variance. 
For $\mathscr{T}$ we take

$$
\mathscr{T}\left(\underline{p}, \underline{Y}_{k}\right)=\frac{1}{2} \sum_{k=N_{0}}^{N}\left\|\underline{z}_{k}-H \underline{Y}_{k}\right\|_{R^{-1}}^{2}+\frac{1}{2} \sum_{k=0}^{N-1}\left\|\underline{Y}_{k+1}-\underline{f}\left(\underline{p}, \underline{Y}_{k}\right)-B \underline{u}_{k+1}\right\|_{\left(G Q G^{\top}\right)^{-1}}^{2}
$$

As mentioned earlier, we derive $\underline{p}^{*}$ and $\underline{Y}_{k}^{*}\left(p^{*}\right)$, which minimize $\mathscr{T}\left(\underline{p}, \underline{Y}_{k}\right)$, in two steps. First we determine the minimizing trajectory $\left\{\underline{Y}_{k}^{*}(\underline{p}) \mid k=0,1, \ldots, N\right\}$ for a certain value of $p$. In the second step we select from this class of minimizing trajectories the trajectory parametrized by $p^{*}$, that minimizes $\mathscr{T}$ globally. In this sense, the class of minimizing trajectories acts as a constraint for the minimization of $\mathscr{T}$ with respect to $\underline{p}$. In order to find the minimizing trajectory, equation (20) is rewritten as

$$
\mathscr{T}\left(\underline{p}, \underline{Y}_{k}\right)=\frac{1}{2} \sum_{k=N_{0}}^{N}\left\|\underline{z}_{k}-H \underline{Y}_{k}\right\|_{R^{-1}}^{2}+\frac{1}{2} \sum_{k=0}^{N-1}\left\|G \underline{w}_{k}\right\|_{(G Q G T)^{-1}}^{2}+\sum_{k=0}^{N-1} \underline{v}_{k+1}^{\mathbf{T}}\left[\underline{Y}_{k+1}-\underline{f}\left(\underline{p}, \underline{Y}_{k}\right)-B \underline{u}_{k+1}-G \underline{w}_{k}\right]
$$

where the adjoint states $\left\{\underline{\boldsymbol{v}}_{k} \mid k=1,2, \ldots, N\right\}$ are introduced. At this point the discrete Pontryagin minimum principle may be applied to find a realization of the system noise sequence $w_{k}$. This leads to the requirement that $G \underline{w}_{k}=G Q G^{\mathrm{T}} \underline{v}_{k+1}$. For the minimization of $\mathscr{T}, \partial \mathscr{T} / \partial \underline{Y}_{k}$ and $\bar{\partial} \mathscr{T} / \partial \underline{v}_{k}$ are set equal to zero, so the smoothed state $\underline{Y}_{k}^{*}$ and the adjoint state $\underline{v}_{k}$ satisfy

$$
\begin{aligned}
\underline{Y}_{k+1}^{*} & =\underline{f}\left(\underline{p}, \underline{Y}_{k}^{*}\right)+B \underline{u}_{k+1}+G Q G^{\mathrm{T}} \underline{v}_{k+1}, \quad k=0, \ldots, N-1, \\
\underline{v}_{k} & =F(\underline{p})^{\mathrm{T}} \underline{v}_{k+1}+H^{\mathrm{T}} R^{-1}\left(\underline{z}_{k}-H \underline{Y}_{k}^{*}\right), \quad k=N_{0}, \ldots, N, \\
\underline{v}_{k} & =F(\underline{p})^{\mathrm{T}} \underline{v}_{k+1}, \quad k=1,2, \ldots, N_{0}-1, \\
\underline{v}_{N+1} & =\underline{0}, \\
\underline{Y}_{0}^{*} & =\underline{0} .
\end{aligned}
$$

This is a two-point boundary value problem (TPBVP). Its solution is the trajectory that minimizes $\mathscr{T}$ for a certain value of the parameter $\underline{p}$. The second part of the estimation procedure consists of finding the minimum of $\mathscr{T}$ with respect to $\underline{p}$.

\subsection{Parameter estimation}

The parameter estimation itself is performed by a gradient-based algorithm, where the gradient of $\mathscr{T}\left(\underline{p}, \underline{Y}_{k}\right)$ with respect to $\underline{p}$ is determined by the method of Chavent. ${ }^{6}$ In trying to find $p^{*}$ we are minimizing $\mathscr{T}\left(\underline{p}, \underline{Y}_{k}\right)$ under the restriction that equations (22)-(26) (the TPBVP) are satisfied. Then $p^{*}$ can be estimated iteratively using a quasi-Newton method. Therefore, we need, besides $\nabla \mathscr{T}(p)$, ipformation about the Hessian $H(p)$ of $\mathscr{T}(\cdot)$ to be able to approximate $\mathscr{T}(\cdot)$ locally by a quadratic function $\mathscr{T}^{\mathbf{q}}(\Delta \underline{p})$ :

$$
\mathscr{T}^{\mathrm{q}}(\Delta \underline{p})=\mathscr{T}(\underline{p})+\Delta \underline{p}^{\mathrm{T}} \nabla \mathscr{T}(\underline{p})+\frac{1}{2} \Delta \underline{p}^{\mathrm{T}} H(\underline{p}) \Delta \underline{p} .
$$

The value for $\Delta \underline{p}$ that minimizes $\mathscr{T}^{\mathrm{q}}$ is found from $\nabla \mathscr{T}^{\mathrm{q}}=\underline{0}$ or $\Delta \underline{p}=-H^{-1}(p) \nabla \mathscr{T}(p)$.

In general, the computation of $H^{-1}(p)$ is replaced by a recursive update of this matrix because of the large amount of computation time required by an exact calculation. In our case, the BFGS-method is used to accomplish this. ${ }^{9}$ It makes the convergence of the iteration almost second order, and it can be proved that if the function $\mathscr{T}(\cdot)$ is purely quadratic with respect to the parameter $\underline{p}$, the number of iterations equals the dimension of $p$ plus one. Furthermore, it is widely accepted that this method is superior for parameter estimation problems with a dimension 


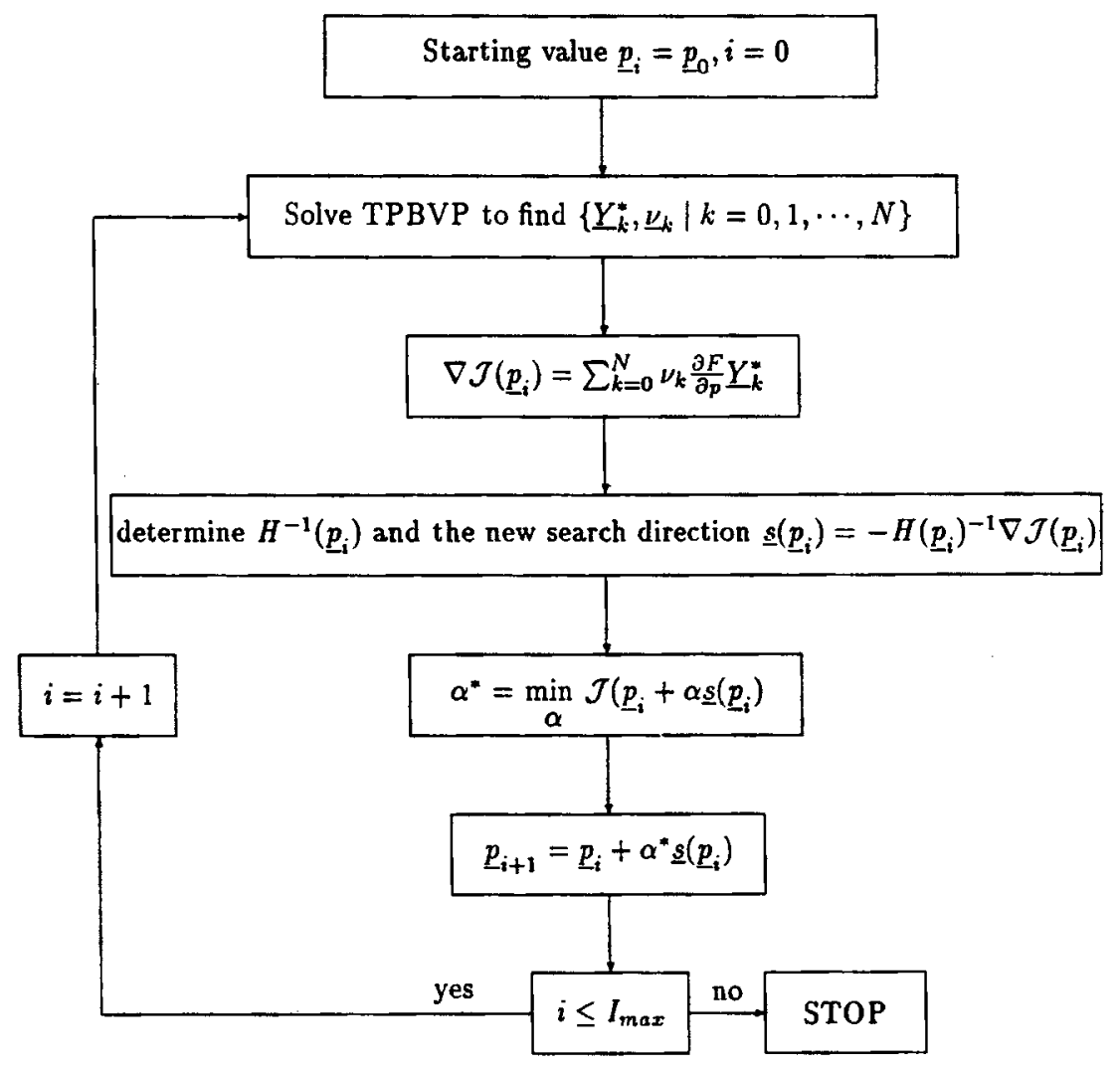

Figure 2. The parameter estimation procedure

of $p$ less than 50 (see Reference 10). The whole procedure is shown schematically in Figure 2. The method of deriving grad $\mathscr{T}$ is described below (see References 6,11).

1. Introduce the Lagrange multipliers $\underline{\xi}_{k}$ and $\underline{\zeta}_{k}$ in order to extend the criterion $\mathscr{T}$,

$$
\begin{aligned}
\mathscr{T}\left(\underline{p}, \underline{Y}_{k}\right)= & \frac{1}{2} \sum_{k=N_{0}}^{N}\left\|\underline{z}_{k}-H \underline{Y}_{k}\right\|_{R^{-1}}^{2}+\frac{1}{2} \sum_{k=0}^{N-1}\left\|G Q G^{\mathrm{T}} \underline{v}_{k+1}\right\|_{(G Q G)}^{2} \mathrm{~T}-1 \\
& +\sum_{k=0}^{N-1} \underline{\xi}_{k+1}^{\mathrm{T}}\left[\underline{Y}_{k+1}-\underline{f}\left(\underline{p}, \underline{Y}_{k}\right)-B \underline{u}_{k+1}-G Q G^{\mathrm{T}} \underline{v}_{k+1}\right] \\
& +\sum_{k=0}^{N_{0}-1} \underline{\zeta}_{k}^{\mathrm{T}}\left[\underline{v}_{k}-F(\underline{p}) \underline{v}_{k+1}\right]+\sum_{k=N_{0}}^{N} \underline{\zeta}_{k}^{\mathrm{T}}\left[\underline{v}_{k}-F(\underline{p}) \underline{v}_{k+1}-H^{\mathrm{T}} R^{-1}\left(\underline{z}_{k}-H \underline{Y}_{k}\right)\right]
\end{aligned}
$$

2. The method of Chavent considers the effects of infinitesimal changes $\Delta p$ in one of the components of $\underline{p}$ on $\mathscr{T}, \underline{Y}_{k}, \underline{v}_{k}, \underline{\xi}_{k}$ and $\underline{\zeta}_{k}$. This is done by linearizing the expression given above. 


$$
\begin{aligned}
\Delta \mathscr{T}=\frac{\partial \mathscr{T}}{\partial p} \Delta p= & \sum_{k=N_{0}}^{N}-H^{\mathrm{T}} R^{-1} \Delta \underline{Y}_{k}^{\mathrm{T}}\left(\underline{z}_{k}-H \underline{Y}_{k}\right)+\sum_{k=0}^{N-1} \Delta \underline{v}_{k+1}^{\mathrm{T}} G Q G^{\mathrm{T}} \underline{v}_{k+1} \\
& +\sum_{k=0}^{N-1} \underline{\xi}_{k+1}^{\mathrm{T}}\left[\Delta \underline{Y}_{k+1}-F(\underline{p}) \Delta \underline{Y}_{k}-G Q G^{\mathrm{T}} \Delta \underline{v}_{k+1}\right] \\
& +\sum_{k=0}^{N-1} \Delta \underline{\xi}_{k+1}^{\mathrm{T}}\left[\underline{Y}_{k+1}-\underline{f}\left(\underline{p}, \underline{Y}_{k}\right)-G Q G^{\mathrm{T}} \underline{v}_{k}\right] \\
& +\sum_{k=0}^{N_{0}-1} \underline{\zeta}_{k}^{\mathrm{T}}\left[\Delta \underline{v}_{k}-F(\underline{p})^{\mathrm{T}} \Delta \underline{v}_{k+1}\right]+\sum_{k=0}^{N_{0}-1} \Delta \underline{\zeta}_{k}^{\mathrm{T}}\left[\underline{v}_{k}-F(\underline{p})^{\mathrm{T}} \underline{v}_{k+1}\right] \\
& +\sum_{k=N_{0}}^{N} \underline{\zeta}_{k}^{\mathrm{T}}\left[\Delta \underline{\underline{y}}_{k}-F(\underline{p})^{\mathrm{T}} \Delta \underline{v}_{k+1}+H^{\mathrm{T}} R^{-1} H \Delta \underline{Y}_{k}\right] \\
& +\sum_{k=N_{0}}^{N} \Delta \underline{\zeta}_{k}^{\mathrm{T}}\left[\underline{y}_{k}-F(\underline{p})^{\mathrm{T}} \underline{\underline{v}}_{k+1}-H^{\mathrm{T}} R^{-1}\left(\underline{z}_{k}-H \underline{Y}_{k}\right)\right] \\
& +\sum_{k=0}^{N-1} \underline{\xi}_{k+1}^{\mathrm{T}} \frac{\partial F}{\partial p} \underline{Y}_{k} \Delta p-\sum_{k=0}^{N} \underline{\zeta}_{k}^{\mathrm{T}} \frac{\partial F^{\mathrm{T}}}{\partial p} \underline{v}_{k+1} \Delta p .
\end{aligned}
$$

3. In equation (29) the terms with $\Delta \xi_{k}$ and $\Delta \zeta_{k}$ can be neglected because the factors between brackets are the left-hand sides of equations (22)-(24) which are equal to zero. Then, by rearranging the remaining terms, one finds

$$
\begin{aligned}
\Delta \mathscr{T}=\frac{\partial \mathscr{T}}{\partial p} \Delta p= & \sum_{k=1}^{N_{0}-1} \Delta \underline{Y}_{k}^{\mathrm{T}}\left[\underline{\xi}_{k}-F(\underline{p}) \underline{\xi}_{k+1}\right] \\
& +\sum_{k=N_{0}}^{N} \Delta \underline{Y}_{k}^{\mathrm{T}}\left[\underline{\xi}_{k}-F(\underline{p}) \underline{\xi}_{k+1}-H^{\mathrm{T}} R^{-1}\left(\underline{z}_{k}-H \underline{Y}_{k}-H \underline{\zeta}_{k}\right)\right] \\
& +\sum_{k=1}^{N} \Delta \underline{v}_{k}^{\mathrm{T}}\left[\underline{\zeta}_{k}-F(\underline{p}) \underline{\zeta}_{k-1}-G Q G^{\mathrm{T}}\left(\underline{\xi}_{k}-H \underline{v}_{k}\right)\right] \\
& +\sum_{k=0}^{N-1} \underline{\xi}_{k+1}^{\mathrm{T}} \frac{\partial F}{\partial p} \underline{Y}_{k} \Delta p+\sum_{k=0}^{N} \underline{\zeta}_{k}^{\mathrm{T}} \frac{\partial F^{\mathrm{T}}}{\partial p} \underline{v}_{k+1} \Delta p .
\end{aligned}
$$

4. The expressions under the summation signs are similar to equations (22)-(24), including the boundary conditions, except that $\xi_{k}$ now plays the role of $\underline{v}_{k}$ whereas $\zeta_{k}$ can be associated with $\underline{Y}_{k}$. In looking for a simple expression for $\operatorname{grad} \mathscr{T}$, we can exploit this similarity. If $\underline{\zeta}_{k}=\underline{0}$ and $\underline{\xi}_{k}=\underline{v}_{k}$ for all $k$, then

$$
\frac{\partial \mathscr{T}}{\partial p}=-\sum_{k=0}^{N} \underline{v}_{k+1}^{\mathrm{T}} \frac{\partial F}{\partial p} \underline{Y}_{k} .
$$

This implies that $\nabla \mathscr{T}$ is simply derived from the solution of the TPBVP, equations (22)-(26), or, with a slightly different interpretation, that the only problem for the simultaneous estimation of states and parameters is finding the solution of this TPBVP.

The fact that the condition for $\underline{Y}^{*}$ is given for the imitial time and the condition for $\underline{v}$ for the final time eliminates the possibility of finding a solution of equations (22)-(24) in a direct way. Although many methods for solving TPBVPs are available in the literature, none of them seems 
very appropriate in our case, because they require excessive computational loads. Therefore, it is necessary to develop an alternative strategy that makes use of the special characteristics of the problem in order to find a procedure which is also applicable to large-scale problems.

\subsection{Solution of the TPBVP}

In this section, a strategy for deriving the solution of the TPBVP is described for linear tidal models where the random input for the open boundaries is modelled by an AR(1)-model:

$$
\begin{aligned}
\underline{Y}_{k+1} & =F(\underline{p}) \underline{Y}_{k}+B \underline{u}_{k+1}+G \underline{w}_{k+1}, \\
\underline{z}_{k} & =H \underline{Y}_{k}+\underline{v}_{k} .
\end{aligned}
$$

Fundamental to the alternative strategy for solving the TPBVP is the fact that the components of $G Q G^{\mathbf{T}} \underline{y}_{k+1}$ in equation (22) only contribute to $\underline{Y}_{k+1}$ through the components $q_{i}^{k+1}$. This means that the smoothed states follow directly from equation (22) once the smoothed boundary is known. The second crucial point is the linearity of the model. This property implies the equality of the mean, which can be found by the Kalman filter, and the mode, the maximum of the likelihood function. Using a Kalman filter, the observations taken from the actual system and modelled by relation (33) can be combined with the information provided by the system model, equation (32), in order to obtain an optimal estimate of the state of the system. If $\hat{\underline{Y}}(k \mid l)$ is defined as the least-squares estimate of $\underline{Y}_{k}$ based on the measurements $\left\{\underline{z}_{1}, \ldots, \underline{z}_{l}\right\}$, and $P(k \mid l)$ represents the covariance matrix of the estimation error, the recursive filter equations to obtain these quantities can be summarized as follows. The optimal state estimate is propagated from measurement time $k-1$ to measurement time $k$ by the equations

$$
\begin{aligned}
& \underline{\hat{Y}}(k \mid k-1)=F(\underline{p}) \underline{\hat{Y}}(k-1 \mid k-1)+B \underline{u}_{k}, \\
& P(k \mid k-1)=F(\underline{p}) P(k-1 \mid k-1) F(\underline{p})^{\mathbf{T}}+G Q G^{\mathrm{T}} .
\end{aligned}
$$

At time $k$, the observation $\underline{z}_{k}$ becomes available. The estimate is updated by the equations

$$
\begin{aligned}
& \underline{\hat{Y}}(k \mid k)=\underline{\hat{Y}}(k \mid k-1)+K(k)\left[\underline{z}_{k}-H \underline{\hat{Y}}(k \mid k-1)\right], \\
& P(k \mid k)=P(k \mid k-1)-K(k) H P(k \mid k-1),
\end{aligned}
$$

where

$$
K(k)=P(k \mid k-1) H^{\mathrm{T}}\left[H P(k \mid k-1) H^{\mathrm{T}}+R\right]^{-1}
$$

is the filter gain. Since the model, equations (32) and (33), is time-invariant, this filter gain will also become time-invariant. ${ }^{12,13}$

The recursive Kalman filter can now be used to determine the solution of the TPBVP by the following steps:

1. Introduce a new state $\underline{Y}_{k}^{\prime}=\left(\underline{Y}_{k}, q_{i}^{k-1}, q_{i}^{k-2}, \ldots, q_{i}^{0}\right)^{\mathrm{T}}$, which contains the history of the stochastic processes $q_{i}$ from the starting time until the present time $k$.

2. Use a Kalman filter to process the data $\left\{\underline{z}_{l} \mid l=N_{0}, \ldots, N-1, N\right\}$ to produce, at the final time $k=N$, the filtered estimate of the state $\underline{Y}^{\prime}(N \mid N)=\left[\underline{Y}(N \mid N), q_{i}(N \mid N), q_{i}(N-1 \mid N), \ldots\right.$, $\left.q_{i}(0 \mid N)\right]^{\mathrm{T}}$. As a consequence the minimum variance realization of $\left\{\underline{w}_{k}, k=1, \ldots, N\right\}$ is found.

3. Finally, solve equations (22)-(24) by substituting $G Q G^{\top} \underline{v}_{k+1}=G \underline{w}_{k}$.

In practice, the whole history of the stochastic processes $\left\{q_{i}^{l} \mid l=0,1, \ldots, k-1, k\right\}$ is not included in $\underline{Y}_{k+1}^{\prime}$ but only the limited history $\left\{q_{i}^{l} \mid l=k-k_{0}, k-k_{0}+1, \ldots, k-1, k\right\}$. The length of this time $\operatorname{lag} k_{0}$ is chosen so that $\left\{q_{i}^{k-k_{0}} \mid i=1, \ldots, i_{\max }\right\}$ has no further influence on the state $\underline{Y}_{k}$. 
This is a direct consequence of the fact that the dynamic equations are hyperbolic, implying that every signal entering the model has only a limited residential time.

The high dimensionality of the Kalman filter equations can be avoided by using a discrete form of the Chandrasekhar-type algorithm. This algorithm was first proposed by Morf et al. ${ }^{14}$ and has been used in numerous applications. It uses the fact that for certain initial conditions the incremental covariance has rank $p$ ( $p$ is the dimension of the system noise process) and can be factorized as follows:

$$
P(k \mid k)-P(k-1 \mid k-1)=S(k) L(k) S(k)^{\mathbf{T}},
$$

where $P(k \mid k)$ is the $n \times n$ covariance matrix of the state estimate at time $k$ based on the observations available up to and including time $k$ ( $n$ is the dimension of the system state). $S(k)$ is an $n \times p$ matrix, and $L(k)$ is a $p \times p$ matrix. For the model, equations (32) and (33), the recursive equations for obtaining the steady-state filter gain are ${ }^{12}$

$$
\begin{aligned}
Y(k+1) & =F(\underline{p}) S(k), \\
M(k+1) & =M(k)+Y(k+1) L(k) Y(k+1)^{\mathrm{T}} H^{\mathrm{T}}, \\
R^{\varepsilon}(k+1) & =R^{\varepsilon}(k)+H Y(k+1) L(k) Y(k+1)^{\mathrm{T}} H^{\mathrm{T}}, \\
K(k+1) & =G(k+1) R^{\varepsilon}(k+1)^{-1} \\
S(k+1) & =Y(k+1)-K(k+1) H Y(k+1), \\
L(k+1) & =L(k)+L(k) Y(k+1)^{\mathrm{T}} H^{\mathrm{T}} R^{\varepsilon}(k)^{-1} H Y(k+1) L(k),
\end{aligned}
$$

where $K(k)$ is the filter gain at time $k, Y(k), M(k)$ and $R^{\varepsilon}(k)$ are factor matrices and the initial condition for the recursion is given by

$$
\begin{aligned}
Y(1) & =G, & M(0) & =0, \\
R^{\ell}(0) & =R, & L(0) & =Q .
\end{aligned}
$$

Equations (40)-(45) are iterated until

$$
\|K(k+1)-K(k)\|<\varepsilon\|K(k)\|,
$$

where $\varepsilon$ is prespecified. Since the underlying deterministic model is of the hyperbolic type, the number of iterations depends on the travelling times of the waves in the model and therefore on the size of the domain of the problem.

\subsection{Extension to non-linear models}

As we have seen, the computational load is a severe restriction when solving the TPBVP. This implies that a compromise has to be found which is applicable to operational models while incorporating the non-linear character of the dynamics as far as possible. So far, we have been able to profit from the fact that solutions of hyperbolic models are determined by the boundaries. This reduced the smoothing problem to the determination of the smoothed boundary. This observation holds for the linear as well as the non-linear case. For large-scale models, the location of the open boundaries is chosen with care. For example, the open boundaries of a shallow sea model are often located in relatively deep water. This implies that the propagation of the tidal waves from the open boundaries onto the model is a rather linear process. So, under the assumption that some data observation points are located in this 'linear part' of the model, smoothed open boundaries can be determined by processing the data by a linear filter. Therefore, they can be determined without drastically violating the dynamics. The proposed method is 
therefore the same as for linear models. Hence, non-linearities in the dynamics can be introduced with very few additional computational requirements.

\subsection{Deterministic parameter estimation problems}

If we assume that, except for the uncertain parameters, the model and the open boundary conditions are perfect, the parameter estimation problem becomes deterministic. In this case the TPBVP can be solved easily without introducing a Kalman filter. In fact the model and its adjoint are now decoupled. By one run of the dynamical model and, afterwards, one run of the adjoint model backwards in time, the TPBVP can be solved. In this case the parameter estimation procedure reduces to the well-known approach introduced by Chavent ${ }^{6}$ for deterministic models.

\section{APPLICATION TO A MODEL OF THE CONTINENTAL SHELF}

\subsection{The continental shelf model}

The shallow sea model of the entire continental shelf, CSM-16, is based on the shallow water equations. In spherical co-ordinates they read $^{15}$

$$
\begin{gathered}
\frac{\partial u}{\partial t}+\frac{u}{R \cos \phi} \frac{\partial u}{\partial \theta}+\frac{v}{R \cos \phi} \frac{\partial}{\partial \phi}(u \cos \phi)-2 \omega v \sin \phi+\frac{g u \sqrt{ }\left(u^{2}+v^{2}\right)}{C^{2}(D+h)} \\
+\frac{g}{R \cos \phi} \frac{\partial h}{\partial \theta}+\frac{1}{\rho_{\mathrm{w}} R \cos \phi} \frac{\partial p_{\mathrm{a}}}{\partial \theta}-\frac{\rho_{\mathrm{a}} C_{\mathrm{d}} V_{\theta} \sqrt{ }\left(V_{\theta}^{2}+V_{\phi}^{2}\right)}{\rho_{\mathrm{w}}(D+h)}=0, \\
\frac{\partial v}{\partial t}+\frac{u}{R \cos \phi} \frac{\partial v}{\partial \theta}+\frac{v}{R} \frac{\partial v}{\partial \phi}+\frac{u^{2} t g \phi}{R}+2 \omega u \sin \phi+\frac{g v \sqrt{ }\left(u^{2}+v^{2}\right)}{C^{2}(D+h)} \\
+\frac{g}{R} \frac{\partial h}{\partial \phi}+\frac{1}{\rho_{\mathrm{w}} R} \frac{\partial p_{\mathrm{a}}}{\partial \phi}-\frac{\rho_{\mathrm{a}} C_{\mathrm{d}} V_{\phi} \sqrt{ }\left(V_{\theta}^{2}+V_{\phi}^{2}\right)}{\rho_{\mathrm{w}}(D+h)}=0, \\
\frac{\partial h}{\partial t}+\frac{1}{R \cos \phi} \frac{\partial}{\partial \theta}[(D+h) u]+\frac{1}{R \cos \phi} \frac{\partial}{\partial \phi}[(D+h) v \cos \phi]=0,
\end{gathered}
$$

where $\phi$ is the northern latitude, $\theta$ the western latitude, $R$ the radius of the earth $\left(6.378 \times 10^{6} \mathrm{~m}\right)$ $V_{\phi}, V_{\theta}$ wind velocities in the $\phi$ and $\theta$ directions and the other symbols have the same meaning as in Section 2.

The spatial domain of approximately $2.3 \times 10^{6} \mathrm{~km}^{2}$, (see Figure 3) is covered with a numerical grid of about 100 (western latitude, stepsize $\Delta \theta=1 / 4 \mathrm{deg}$.) by 85 (northern latitude, stepsize $\Delta \phi=1 / 6$ deg.) cells. A large number of these are located on the mainland, so the number of active grid cells is reduced to approximately 5000 , which makes the dimension of the state vector approximately 15000 . The ADI-scheme described by Stelling ${ }^{8}$ is used to discretize the dynamic equations (see also Reference 11).

\subsection{Simultaneous estimation of the bottom friction and the depth}

The geometry of a (tidal) flow region is usually derived from nautical cards. One of the purposes of these cards is to guide large ships safely through shallow waters. Therefore, these cards express the shallowness rather than the depth of a flow region. If we use these maps to prescribe the depth, it is reasonable to assume that this prescription of the geometry is erroneous. In this section we include these uncertainties in our parameter estimation problem. The whole computational 


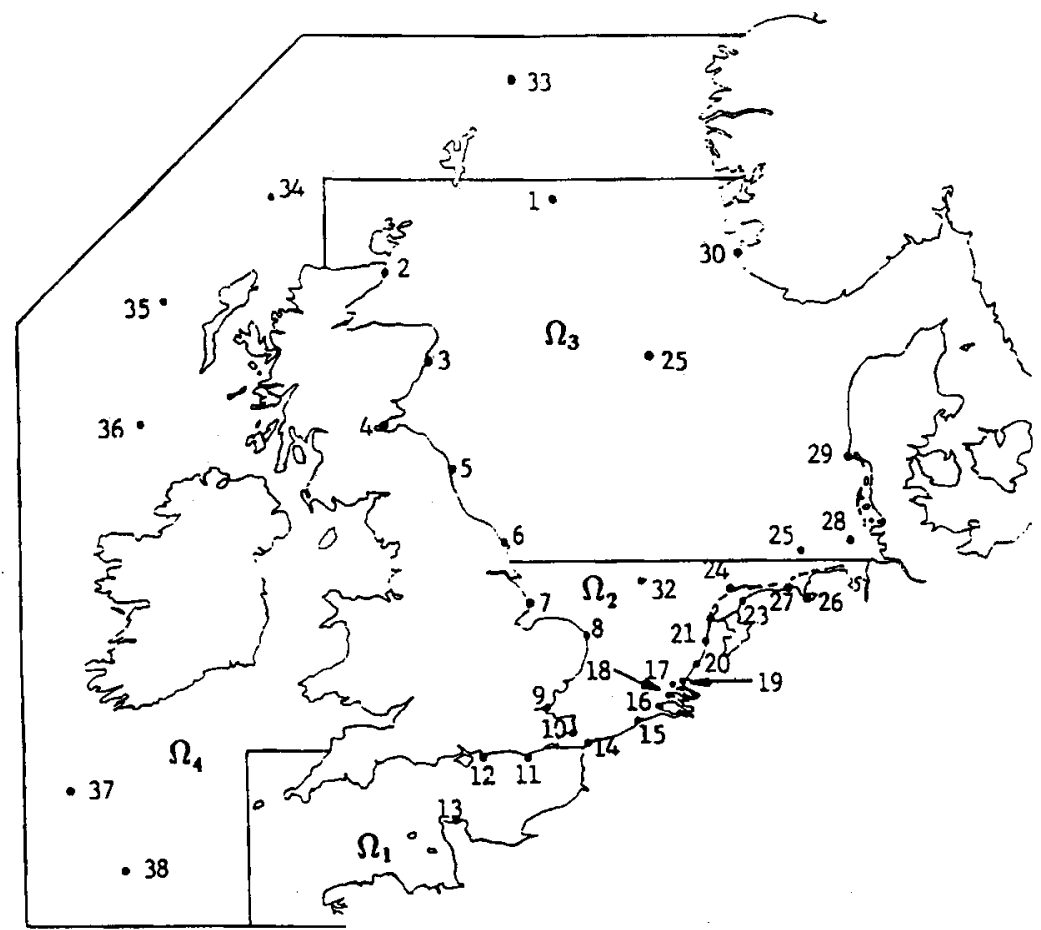

Figure 3. The domain $\Omega$ of CSM-16.

1, J76-55; 2, Wick; 3, Aberdeen; 4, Leith; 5, North Shields; 6, Scarborough; 7, Inner Dowsing; 8, Lowestoft; 9 , Southend; 10, Dover; 11, Newhaven; 12, Portsmouth; 13, Cherbourg; 14, Calais; 15, Oostende; 16, Vlissingen; 17, BG-2; 18, Goeree; 19, H.v. Holland; 20, Scheveningen; 21, IJmuiden; 22, Den Helder; 23, Harlingen; 24, W-Terschelling; 25, Huibertgat; 26, Delfzijl; 27, Borkum; 28, Helgoland; 29, Esbjerg; 30, Stavanger; 31, Ekofisk; 32, Station K13A; 33, S8; 34, S3; 35, S1; $36, \mathrm{~S} 14 ; 37, \mathrm{C} 5 ; 38, \mathrm{C} 3$

domain $\Omega$ is divided into a number of disjunct subdomains $\Omega_{j}^{D}, j=1, \ldots, j_{\max }$. For each subdomain $\Omega_{j}^{D}$, a parameter $d_{j}$ is estimated to adjust the mean level of the depth in the subdomain $\boldsymbol{\Omega}_{j}$

$$
D_{m, n}=D_{m, n}^{M}+d_{j} \quad \text { if } \quad(m, n) \in \Omega_{j}^{D},
$$

with $D_{m, n}^{M}$ the depth used in the operational model so far. The same strategy can be applied to the adjustment of the bottom friction coefficients in subdomains $\Omega_{i}^{C}, i=1, \ldots, i_{\max }$ :

$$
C_{m, n}=C_{m, n}^{M}+c_{i} \quad \text { if }(m, n) \in \Omega_{i}^{C} .
$$

The subdomains $\Omega_{j}^{D}$ for the adjustment of the depth are not necessarily equal to the domains $\Omega_{i}^{c}$ for the estimation of the friction coefficient $c_{i}$. The reason for choosing different partitions is that the influence on the water levels of adapting the bottom friction differs from the influence of depth adaptation. The bottom friction affects the amplitude of the tide and the phase shift between the water level elevation and the velocities. The depth determines the propagation speed of the long waves and the water volume of the domain $\Omega$. Moreover, the depth is more prominently present in the dynamic equations than the bottom friction. For the minimization process in the parameter space, this implies that we cannot expect the error criterion $\mathscr{T}(p)$ to be purely quadratic with respect to the parameters $c_{i}$ and $d_{j}$, as is approximately the case when only 


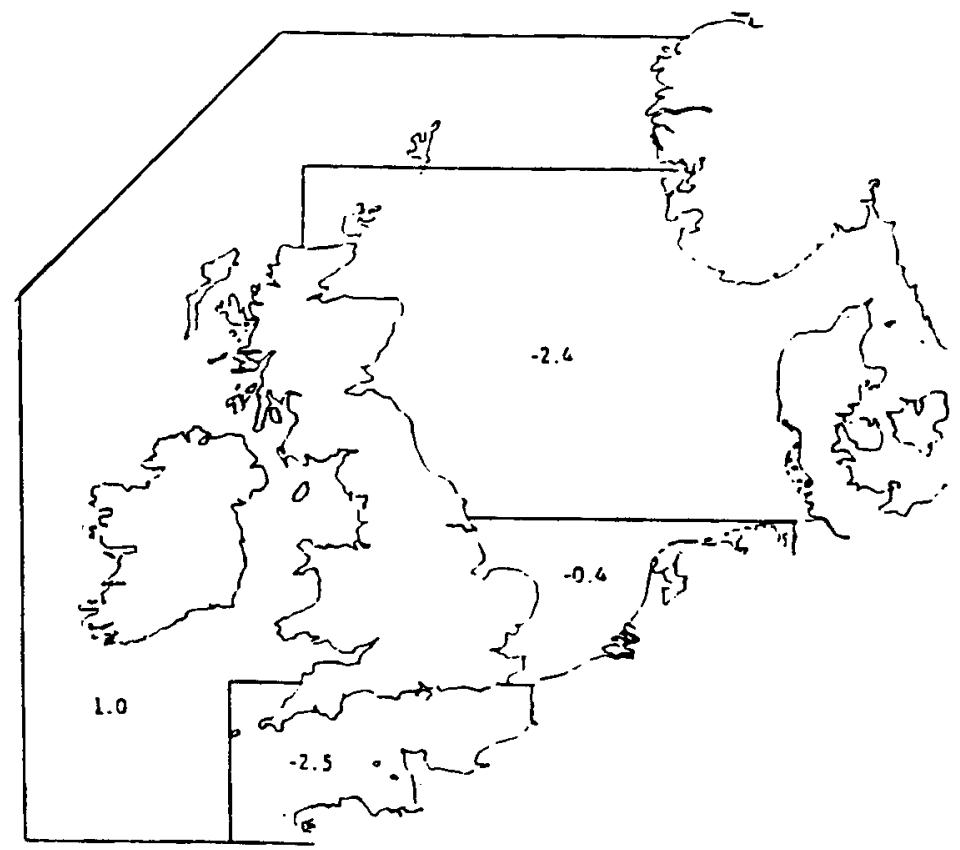

Figure 4. The adjustments of depth (m) found in Experiment I. A negative adjustment means that the depth is reduced compared to the operational model. The black dots denote the data observation points

bottom friction coefficients $c_{i}$ are estimated. This increased non-linearity may hamper the minimization because local minima will be more pronounced.

For the parameter estimation experiments that are discussed in this paragraph, we used field data from ten data points* observed on 12 February 1989. In this period the meteorological influence on the tidal elevation was minimal. Nevertheless, an atmospheric model provided the meteorological input for CSM-16. This implies that the effect of incorrect meteorological inputs on the parameter estimation can be neglected in this case. The successive experiments deal with an increased level of detail in the choice of the partition $\Omega=\cup \Omega_{i}^{D}$ for the estimation of $d_{j}$ while the partition $\Omega=\cup \Omega_{i}^{C}$ for the estimation of $c_{l}$ remains the same. We now briefly comment on the results of the experiments, and give some general conclusions.

Experiment I. For the first experiment we take $\Omega_{j}^{D}=\Omega_{j}^{C}$ for $j=1, \ldots, 4$ (see Figure 4). Compared to the operational model, the value of the error criterion $\mathscr{T}$ is decreased from 45.79 to 32.44 within eight iterations (Figure 5). This $29 \%$ decrease in $\mathscr{T}$ causes an average reduction of $16 \%$ in the RMS error in the data observation points. The RMS errors in the data observation points are given in Column 2 of Table $I$.

Experiment $I I$. The subdomains $\Omega_{j}^{D}, j=1, \ldots, 4$, are chosen without requiring uniformity of the depth in each subdomain. From a physical point of view, the estimation of a $d_{j}$ in subdomains

* Wick, North Shields, Lowestoft, Southend, Newhaven, Dover, Vlissingen, Hoek van Holland, IJmuiden and Den Helder (see Figure 3). 


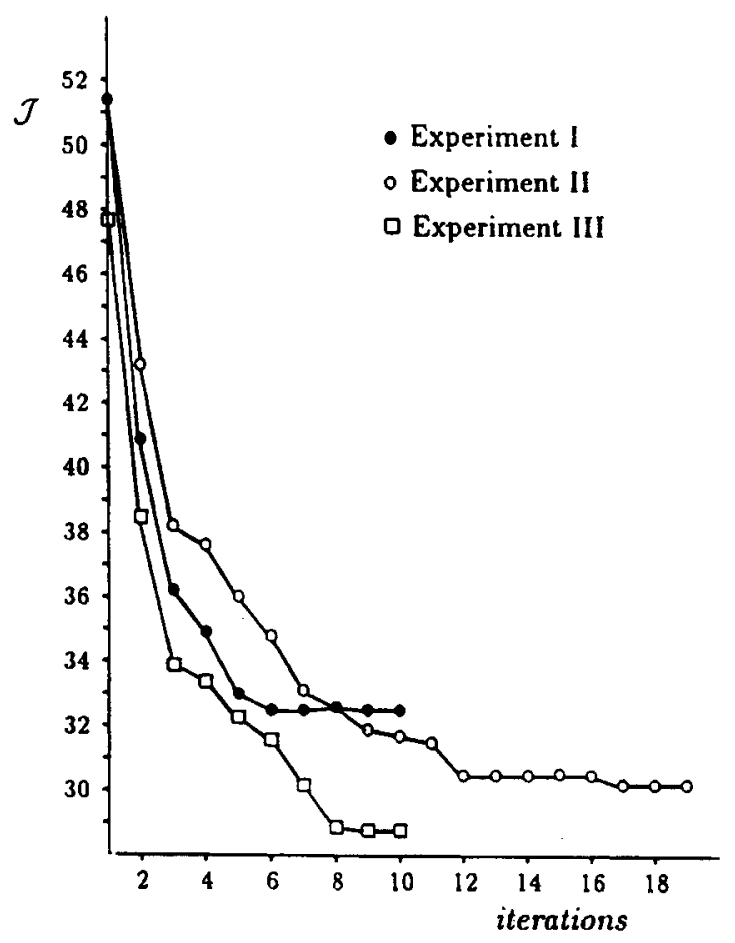

Figure 5. The reduction of the output least-squares function

Table I. The RMS errors [m] in some data observation points

\begin{tabular}{|c|c|c|c|c|}
\hline Station & $\begin{array}{l}\text { Operational } \\
\text { model }\end{array}$ & Experiment I & Experiment II & Experiment IIl \\
\hline Wick & $0-157$ & $0 \cdot 110$ & 0.127 & 0.089 \\
\hline North Shields & $0 \cdot 152$ & $0 \cdot 145$ & $0 \cdot 145$ & 0.115 \\
\hline Lowestoft & $0 \cdot 104$ & 0.083 & 0.095 & 0.076 \\
\hline Dover & $0 \cdot 171$ & 0.147 & 0.131 & 0.144 \\
\hline Vlissingen & $0 \cdot 261$ & $0 \cdot 204$ & 0.211 & 0.200 \\
\hline H.v.Holland & $0 \cdot 124$ & $0 \cdot 118$ & 0.138 & $0 \cdot 117$ \\
\hline IJmuiden & $0 \cdot 157$ & $0 \cdot 133$ & 0.119 & 0.131 \\
\hline Den Helder & $0 \cdot 121$ & $0 \cdot 105$ & 0.096 & $0 \cdot 101$ \\
\hline
\end{tabular}

containing both deep and shallow areas is more or less the same as the estimation of $d_{j}$ in the shallow area. Consequently, a new partition $\Omega=\cup \Omega_{j}^{D}$ that is based on the uniformity of depth in $\Omega_{j}^{D}$ will be more suitable. Another reason to change the partition is that the number of subdomains $\Omega_{j}^{D}$ can be increased for estimating $d_{j}$. It has already been noted that the influence of depth adjustment is far greater than the adjustment of bottom friction coefficients and has a stronger local effect. Therefore, we make a new partition of $\Omega$ with 17 subdomains (see Figure 6). After 12 iteration steps, the value of $\mathscr{T}$ is now reduced to 30.08 (Figure 5). Compared to the operational model this is a reduction of $34 \%$. The RMS errors in various data observation points 


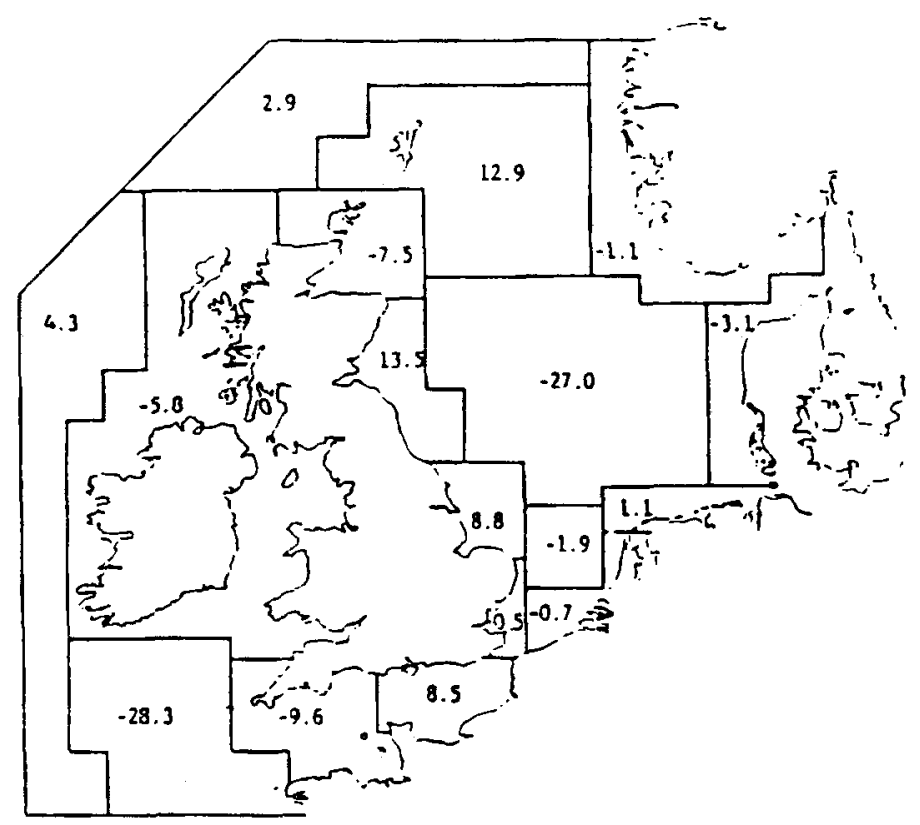

Figure 6. The adjustments of depth (m) found in Experiment II

can be found in Table I, Column 3, and the effects of the parameter estimation are shown in Figures 7-9.

Above we have briefly indicated the effect of various parameter estimation experiments in terms of reduction of $\mathscr{T}$ and the RMS errors. As well as these direct consequences, the behaviour of the estimation method is also an important issue which raises questions concerning the uniqueness of the adjustments, the convergence to local minima, etc. We now consider these aspects point wise.

1. In the final phase of the iteration some parameters are adjusted without reducing the value of $\mathscr{T}$ significantly. This had already been noted for $c_{4}$ in an experiment where only bottom friction coefficients were estimated. It also occurs when simultaneously estimating $d_{j}$ and $c_{i}$.

2. In Experiments I and II, the partitions of $\Omega=\cup \Omega_{i}^{C}$ for the bottom friction coefficient are identical. The differences between the estimated values of $c_{i}, i=1, \ldots, 4$, are minor, especially for $c_{1}, c_{2}$ and $c_{3}$ (Table II). The large (percentage) difference for $c_{4}$ is not surprising given the fact that $c_{4}$ has a very limited influence on the model results.

3. The problem of convergence to local minima has to be studied carefully. Various estimation attempts with different initial conditions resulted in $\mathscr{T}$-values up to 35 . Compared to the $\mathscr{T}$-value of the operational model, $45 \cdot 69$, this is still a substantial improvement. When only bottom friction coefficients were estimated, convergence to local minima could be avoided by normalizing the search direction. ${ }^{11}$ Unfortunately, in the case of a simultaneous estimation of bottom friction and depth this normalization is not capable of avoiding this problem completely. This proves once more that the error criterion $\mathscr{T}(p)$ is not quadratic with respect to the parameters, and the more pronounced local effects of depth adaptation hamper the estimation process. 
4. The adjustments in the southern part of the North Sea are very small. It seems that the depth prescribed for the operational model is already close to optimal.

5. If we consider the adjustments of the parameters, we note that some of them are rather large. For the depth, a margin of 5-10\% is usually assumed to be reasonable. In Experiment II we find the largest adjustments in the subdomains along the east coast of England and south of
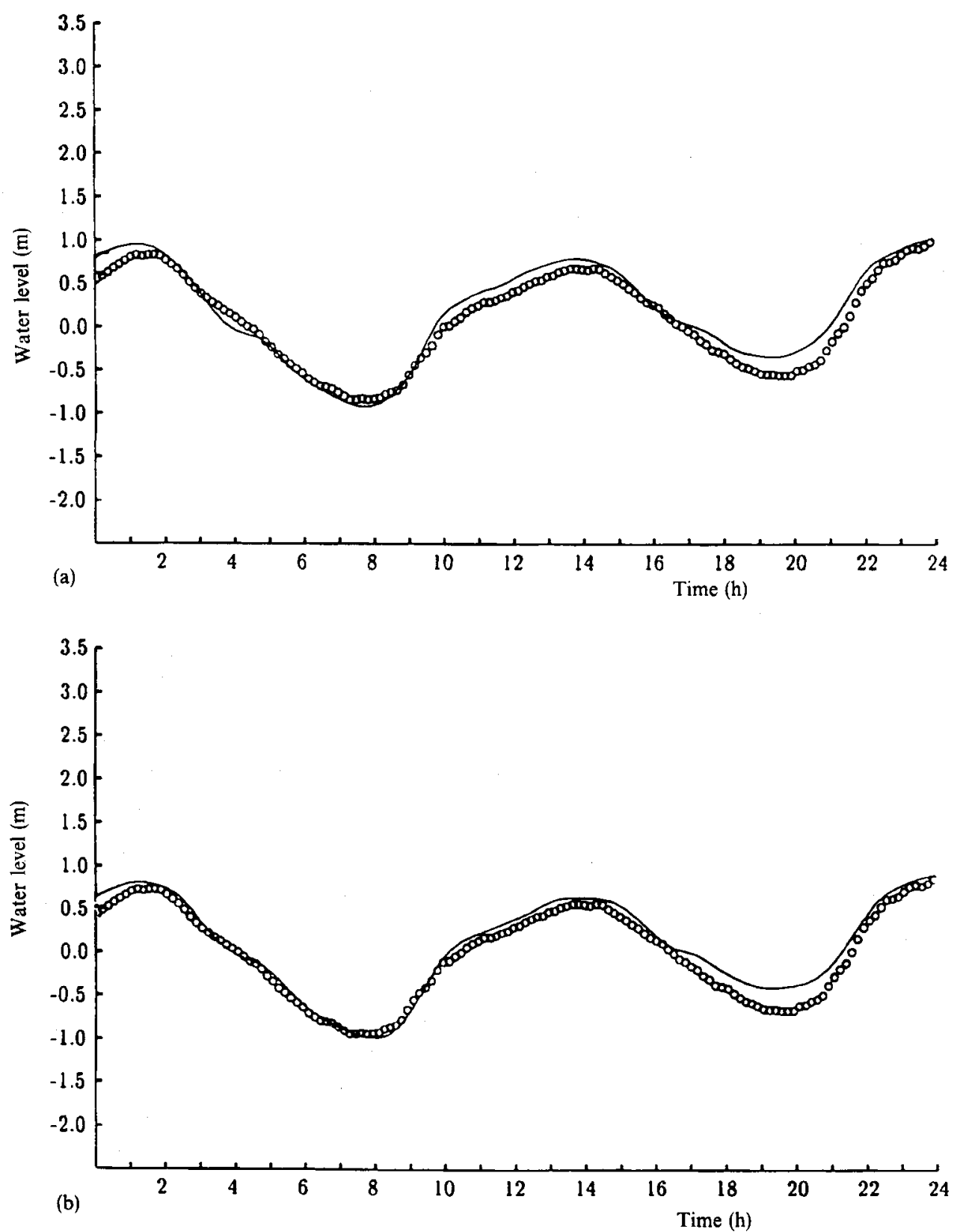

Figure 7. The effects of parameter estimation for the Lowestoft station on 12 February 1989. $00 \bigcirc$, field data; CSM-16. (a) Operational model; (b) Experiment I; (c) Experiment II 


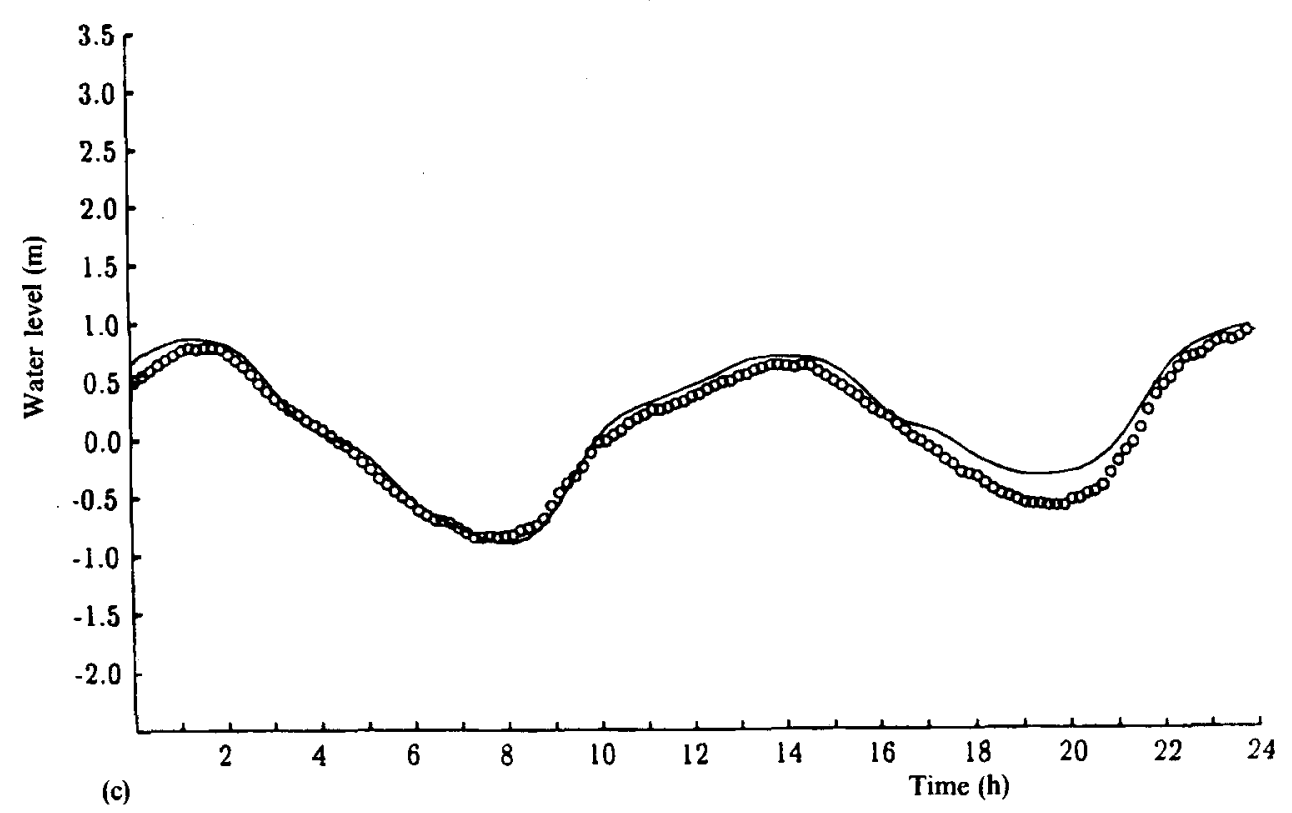

Figure 7. (Continued)

the Channel. Besides the argument already stated in one of the previous points (a large adaptation of a parameter that has almost no effect on the value of the criterion), there is also a physical explanation. The data observation points are concentrated in the southern part of the North Sea (see Figure 4). The parameter estimation procedure searches for a parameter $p^{*}$ that minimizes $\mathscr{T}$, so it will use all its freedom to simulate the water level elevation in this area as closely as possible. The data do not provide enough information to simulate the tidal flow accurately in other parts of the continental shelf. In this way the adjustments of the parameters may be influenced by other model errors that are not explicitly taken into account. If, for example, the open boundary condition is erroneous, the parameters will be adjusted so that the error in the simulated tidal wave is reduced while propagating to the southern part of the North Sea.

6. In order to get an idea of the computation time required, we express it in terms of the number of model simulations. For simplicity, we assume that solving the adjoint model is computationally equivalent to a CSM-16 simulation. So for every iteration step we need (i) a CSM-16 simulation and the solution of the adjoint model, to determine $\nabla \mathscr{T}$, and (ii) two to three CSM-16 simulations to find the line minimum which is, on average, 4.5 model simulations for each iteration step. We let one model simulation cover a period of $4 \mathrm{~d}: 3 \mathrm{~d}$ to remove the transient data due to the initial condition and $1 \mathrm{~d}$ on which the data are compared with the model results. The numerical experiments are performed on a CRAY Y-MP2E/132 computer. The simulation of $1 \mathrm{~d}$ (with a time step $\Delta t=10 \mathrm{~min}$ ) requires approximately $12 \mathrm{~s}$. This leads to $4 \mathrm{~min}$ CPU time for each iteration step. Therefore, it takes approximately $72 \mathrm{~min}$ to estimate the parameters in Experiment II. 


\subsection{Estimation of the wind stress parametrization}

In Section 3.2, the CSM-16 model was calibrated for mild meteorological conditions (i.e. no wind or minor wind force). In this calibration the bottom friction coefficient and the depth of the flow region were adjusted until maximal agreement was obtained between the simulated water levels and the data.
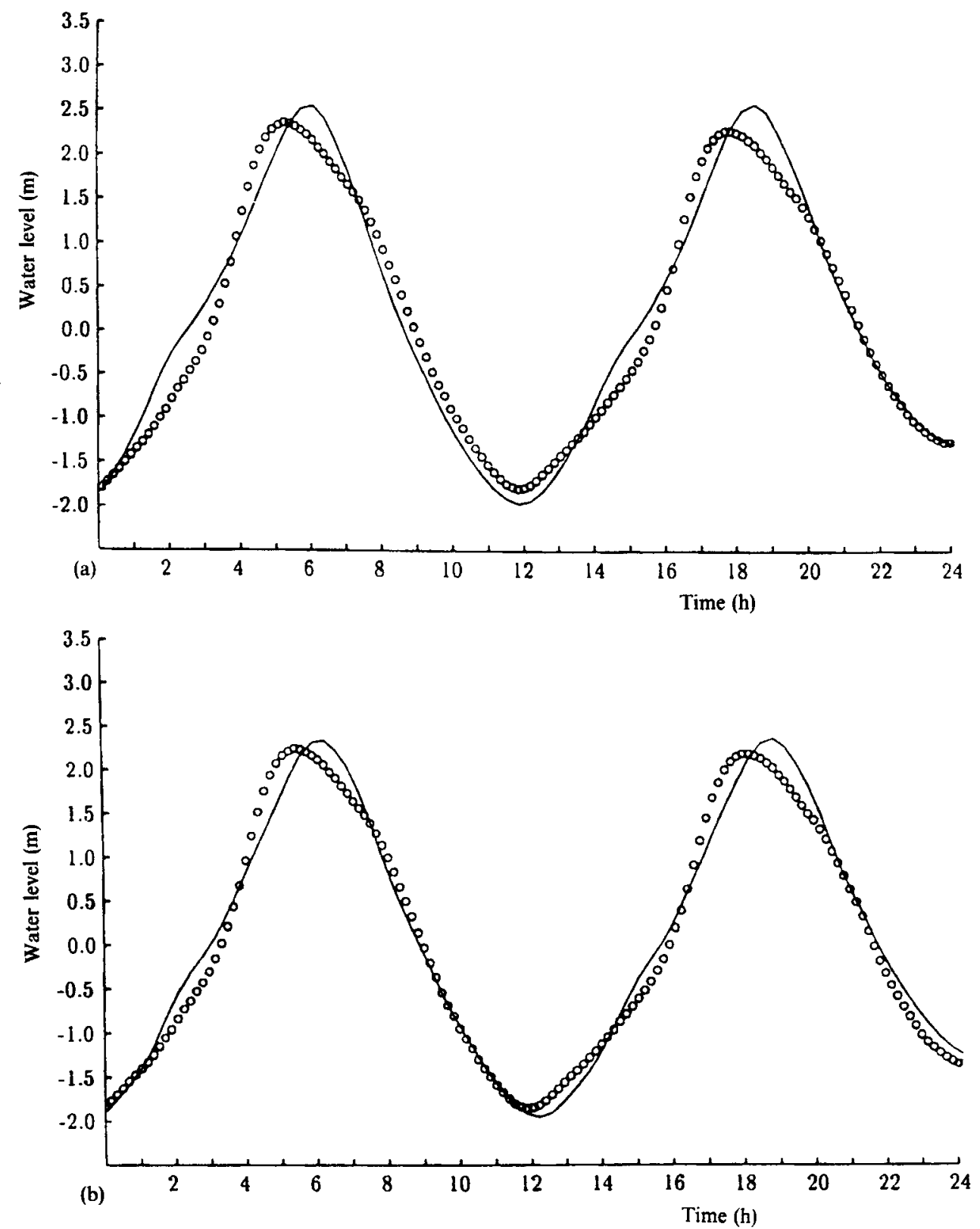

Figure 8. The effects of parameter estimation for the Vlissingen station on 12 February 1989. 000 , Field data; CSM-16. (a) Operational model; (b) Experiment I; (c) Experiment II 


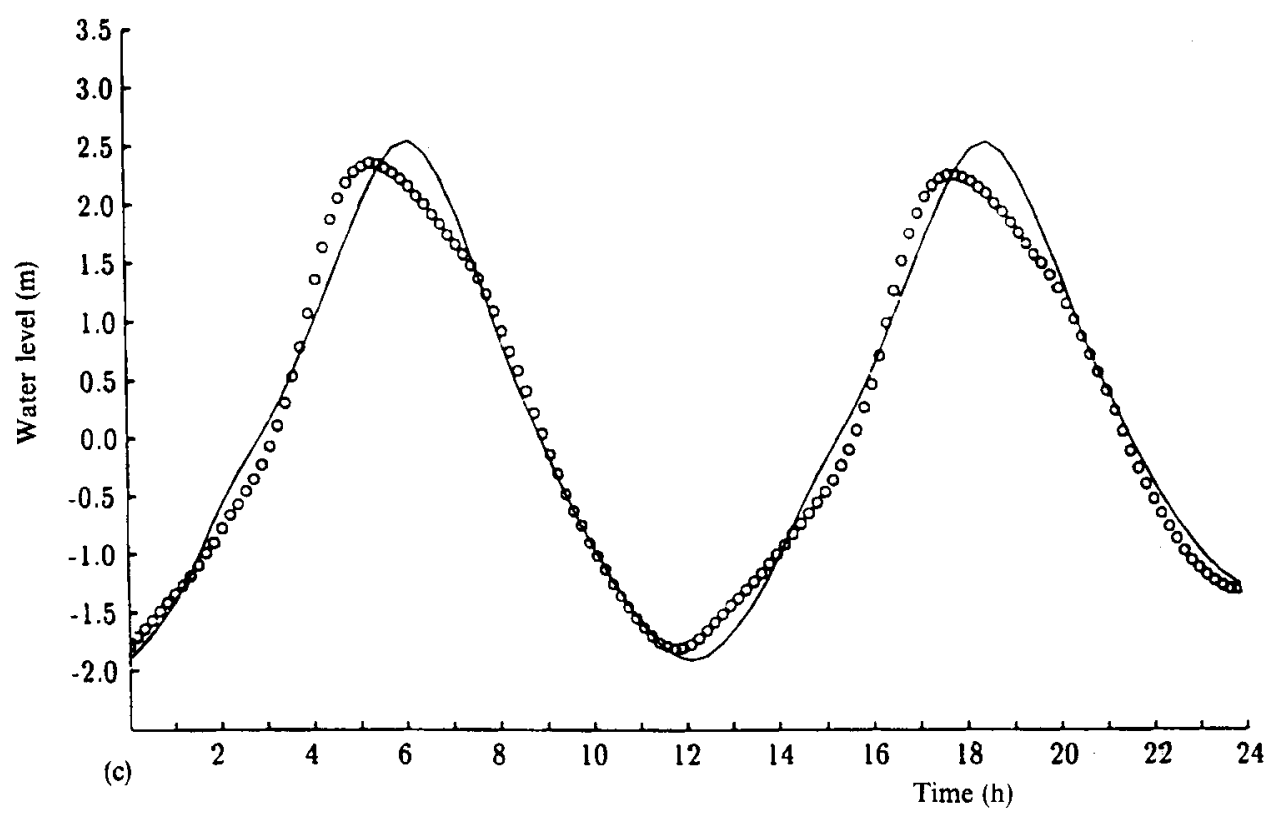

Figure 8. (Continued)

In this section we deal with the calibration of the model under storm conditions. For the North Sea especially, storms have a significant effect on water levels. This is a consequence of the relative shallowness of this area and the effect of its funnel shape on north-western storms. To give an idea of the effect of such storms, surges of the order of 1-2 $\mathrm{m}$ are not exceptional for stations in the Dutch coastal area.

In CSM-16 the wind effect is modelled by a forcing term in the dynamic equations of the velocities. In equations (53) and (54), $C_{\mathrm{d}}$ is the surface drag coefficient. It is a proportionality constant in the relation of the surface shear stress $\tau_{\mathrm{s}}$ and the square of the wind velocity $U_{10}$ (customarily measured at $10 \mathrm{~m}$ above the surface). Thus,

$$
\tau_{\mathrm{s}}=\rho_{\mathrm{a}} C_{\mathrm{d}} U_{10}\left|U_{10}\right| \text {. }
$$

In the literature a wide variety of values have been reported for $C_{\mathrm{d}}$ (see Reference 16 for a recent overview). These $C_{\mathrm{d}}$-values are found from site measurements and show that the drag coefficient is not a constant but tends to increase with the wind speed $U_{10}$. On the basis of scatterplots of $C_{d}$ versus $U_{10}$, it is often suggested that the drag coefficient is a linear function of the wind speed: $C_{\mathrm{d}}=a+b U_{10}$. However, there is a great variability in the values of $a$ and $b$ in this parametrization. It appears that the parameters $a$ and $b$ are regionally dependent. A closer inspection suggests that the drag coefficients for shallow waters such as lakes and estuaries tend to be larger than for deep seas and oceans.

Recent investigations suggest that the surface drag is not properly described as a function of only the wind speed. It is argued that for the formulation of the surface drag it is more realistic to use the state of the air-sea interface. This state is determined by the surface waves which act as roughness elements influencing the momentum flux from the atmosphere to the sea. For further details on this topic, see References 17 and 18.

The present operational version of CSM-16 is still equipped with a 'traditional' $C_{\mathrm{d}}$-parametrization which takes only the wind speed into account. However, this parametrization is slightly 
adapted compared to the linear form mentioned above. It contains four adjustable parameters:

$$
C_{\mathrm{d}}\left(U_{10}\right)\left\{\begin{array}{lll}
y_{1} & \text { if } & U_{10}<x_{1}, \\
y_{1}+\frac{y_{2}-y_{1}}{x_{2}-x_{1}}\left(U_{10}-x_{1}\right) & \text { if } & x_{1} \leqslant U_{10} \leqslant x_{2}, \\
y_{2} & \text { if } & U_{10}>x_{2} .
\end{array}\right.
$$
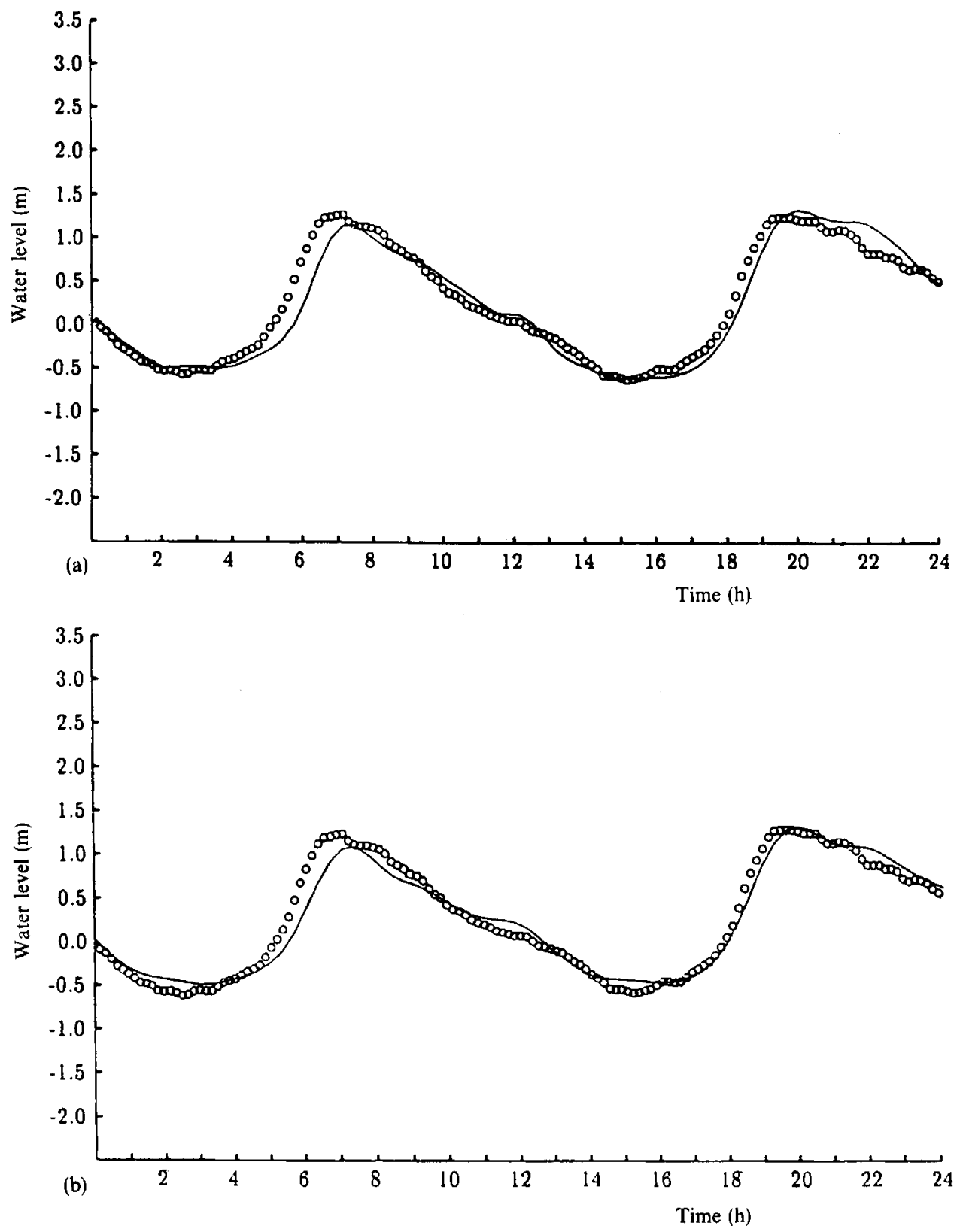

Figure 9. The effects of parameter estimation for the IJmuiden station on 12 February 1989. 000 , field data; CSM-16. (a) Operational model; (b) Experiment I; (c) Experiment II 


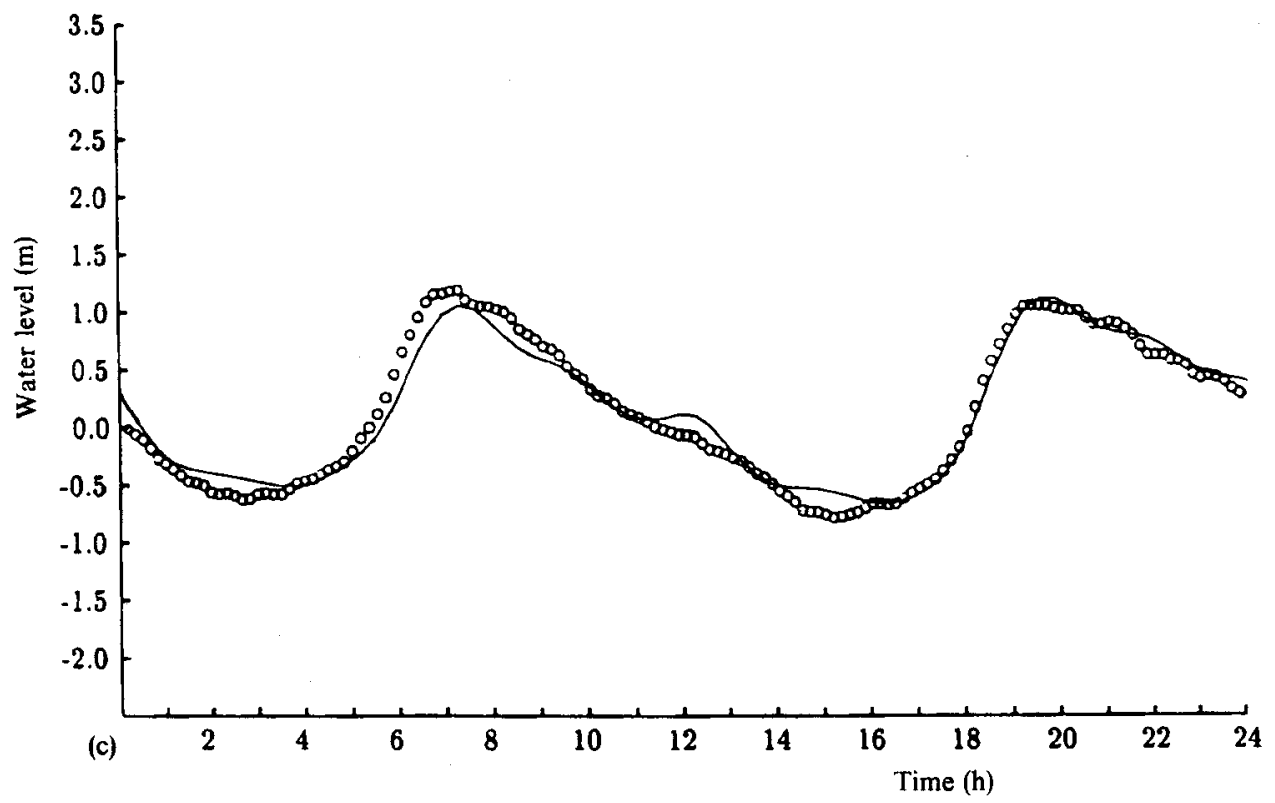

Figure 9. (Continued)

Table II. The adjustments of the bottom friction coefficients (Chezy values)

\begin{tabular}{lcccc}
\hline & $c_{1}$ & $c_{2}$ & $c_{3}$ & $c_{\mathbf{4}}$ \\
\hline Experiment I & 15 & -12 & 7 & -7 \\
Experiment II & 17 & -10 & 8 & -3 \\
\hline
\end{tabular}

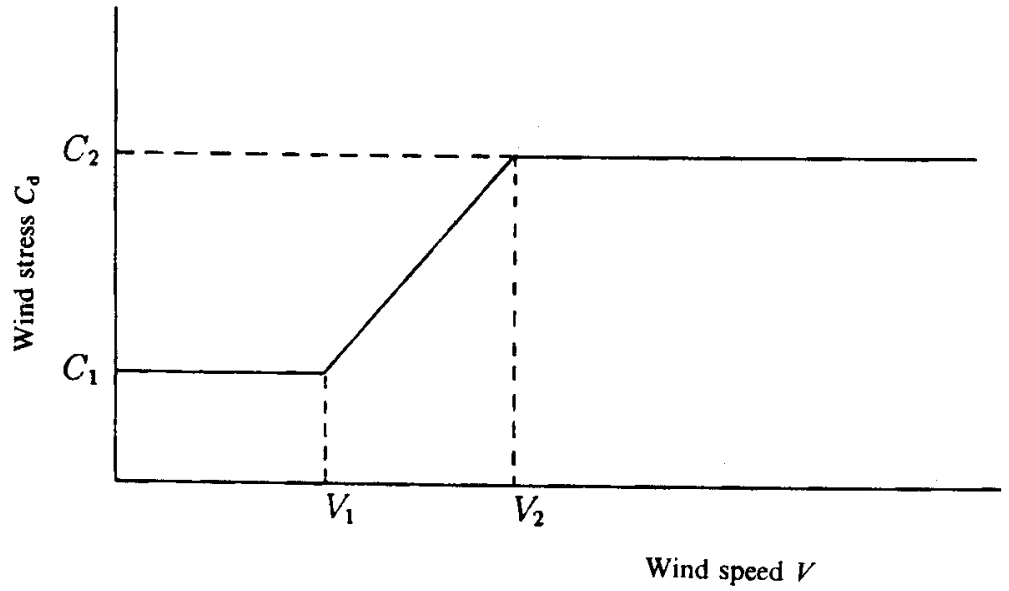

Figure 10. A general $C_{\mathrm{d}^{-}}-V$ curve 
(See Figure 10 for an illustration of $C_{\mathrm{d}}$ as function of wind speed.) In the operational model the following values are used for the parameters: $x_{1}=10 \mathrm{~m} \mathrm{~s}^{-1}, y_{1}=0.001, x_{2}=15 \mathrm{~m} \mathrm{~s}^{-1}$ and $y_{2}=0.0025$. These values were obtained some time ago by a manual calibration of the model.

Given the importance of an accurate prediction of storm surges and the variability in $C_{\mathrm{d}}$ that was reported in the literature, the calibration of the $C_{\mathrm{d}}$ coefficient is repeated for a number of storms. The parameter estimation procedure is applied to identify the $C_{\mathrm{d}}$-parameters $\left(x_{1}, y_{y}, x_{2}, y_{2}\right)$.

The $C_{\mathrm{d}}$-coefficient is calibrated using data from three storm periods: February 1989, February 1983 and November 1981. During these calibration experiments the depth and bottom friction coefficients are not adapted, and the original values of the operational model are used. The results are summarized in Figure 11 (illustration of the optimized $C_{\mathrm{d}^{-}}$-coefficients) and Figure 12 (where observed water levels during the 1983 storm are compared with those according to CSM-16 with the calibrated $C_{\mathrm{d}}$-coefficient).

Two main conclusions can be drawn from these results.

1. The optimized $C_{\mathrm{d}}$-parametrizations of the 1981 and 1989 storms do not differ much from the one used in the operational model (in this comparison the emphasis is laid on the $C_{\mathrm{d}}$-values for wind speeds greater than $10 \mathrm{~m} \mathrm{~s}^{-1}$ since these contribute most significantly to the surface roughness). The $C_{d}$-values found for the 1983 storm turn out to be systematically smaller than the $C_{\mathrm{d}}$-values for the other storms. These results indicate that the parameters in the $C_{\mathrm{d}}$ coefficient are probably not unique and can still depend on the specific storm. On the other hand, the variability of the $C_{\mathrm{d}}$ coefficient can be another indication that this wind-dependent parametrization of the drag coefficient is too simple for an accurate description of the sea surface roughness.

2. In all three optimizations the error criterion $\mathscr{T}$ is only marginally reduced. Apparently the manual calibration performed in the past, and leading to the $C_{\mathrm{d}}$-coefficient of the present operational model, had already given acceptable results for the water levels during storm conditions. On the other hand, it is expected that the error criterion is rather flat in the

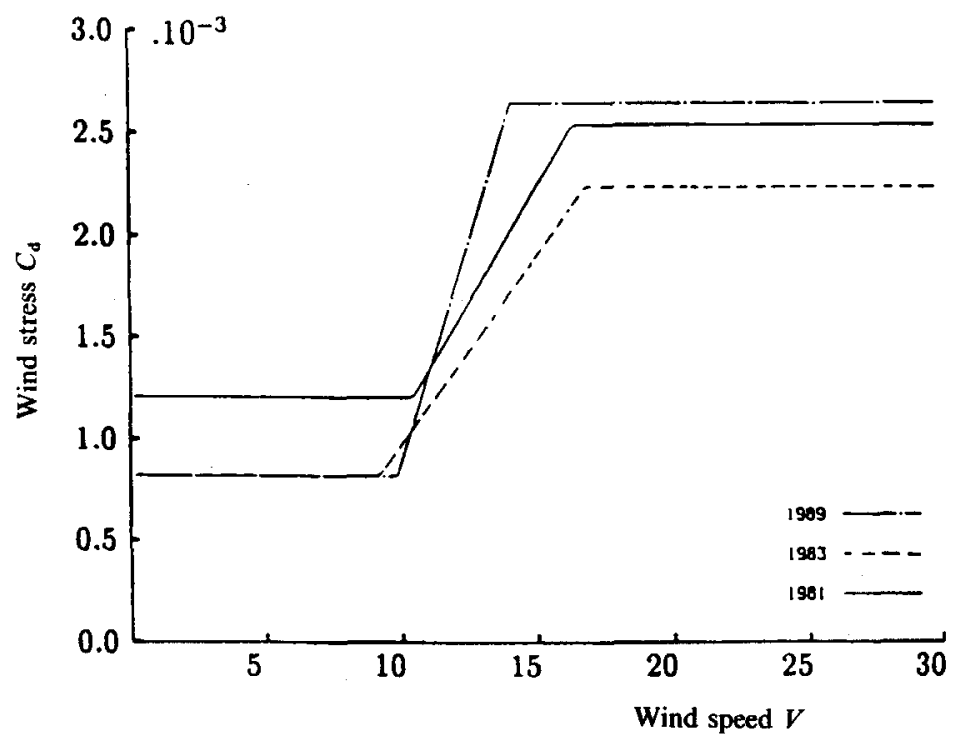

Figure 11. The optimized $C_{\mathrm{d}}-V$ curves for three different storms 


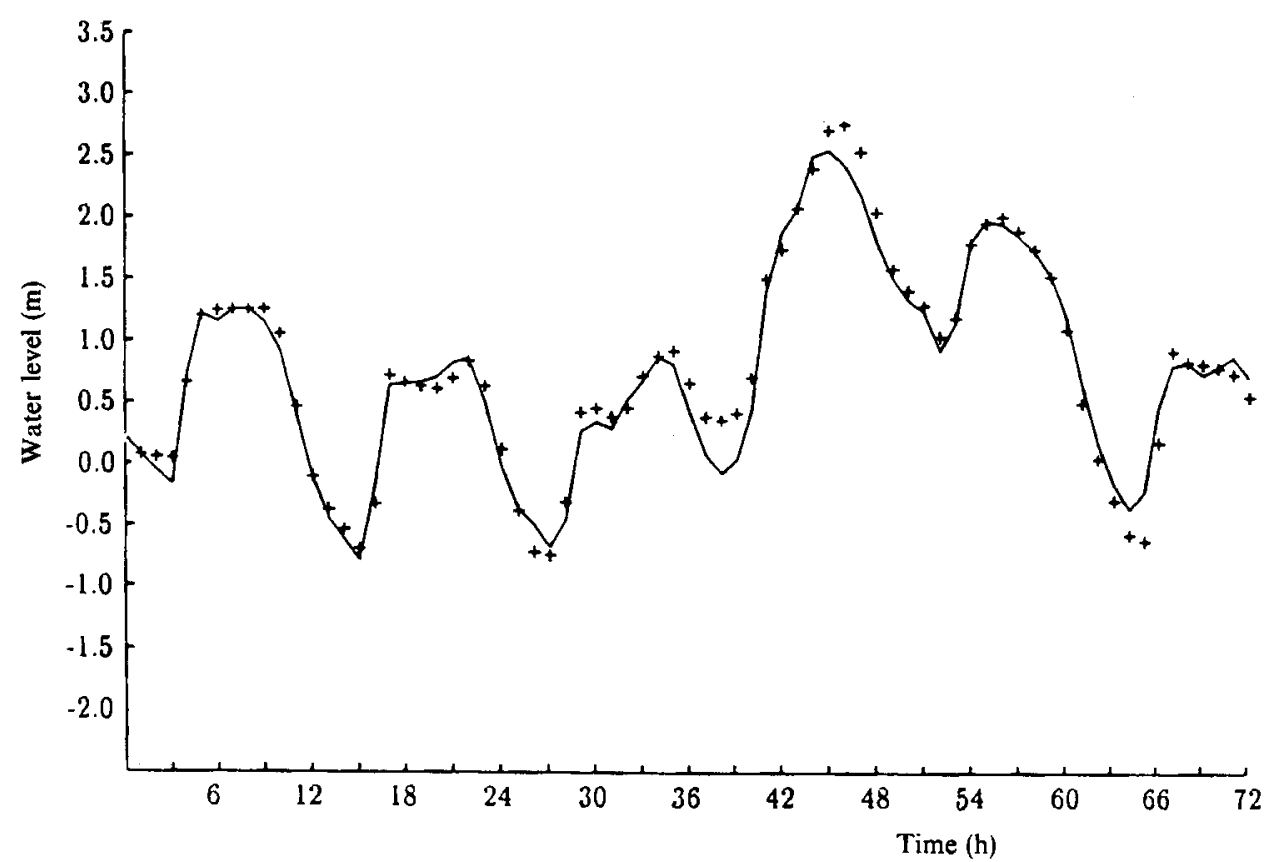

Figure 12. Water levels for the Den Helder station between 31 January and 2 February $1983 .+++$, field data; --, CSM-16. Experiment III.

neighbourhood of the optimized parameters since the reduction of the error criterion is small with respect to the variation in the $C_{\mathrm{d}}$-parameters. This finding is supported by a sensitivity analysis of the model with respect to $\left\{x_{1}, y_{1}, x_{2}, y_{2}\right\}$. This illustrates the model's limited sensitivity for the $C_{\mathrm{d}}$-parameters.

\subsection{Simultaneous estimation of the bottom friction, depth and open boundary conditions}

From the experiments discussed in Section 3.2, we see that the performance of the operational model can be improved by adjusting the bottom friction coefficients and the depth. For some of the estimated parameters, the adjustments are rather large. One possible explanation is that these parameters are adjusted to compensate for other model errors which are not explicitly indicated as uncertainties, such as the uncertainties in the prescription of the open boundary conditions. If we take a closer look at Figure 6, we see that the largest adjustments are found in the area along the east coast of England and south of the Channel. The tidal waves enter the continental shelf at the open boundaries and propagate through these areas before they reach the southern part of the North Sea where most of the data observation points are located. Hence, our interpretation is that the depth is corrected for errors in the prescription of the open boundary error in order to represent the tide in the southern part of the North Sea as closely as possible. For the overall performance of CSM-16 and a sound physical interpretation, it might be better to take these boundary errors explicitly into account. This can be done by introducing a stochastic boundary forcing.

Experiment III. The uncertainty in the open boundaries is represented by a number of stochastic processes, $q_{i}$, satisfying AR(1) models.

$$
q_{i}^{k+1}=\rho q_{i}^{k}+\eta_{i}^{k+1} .
$$




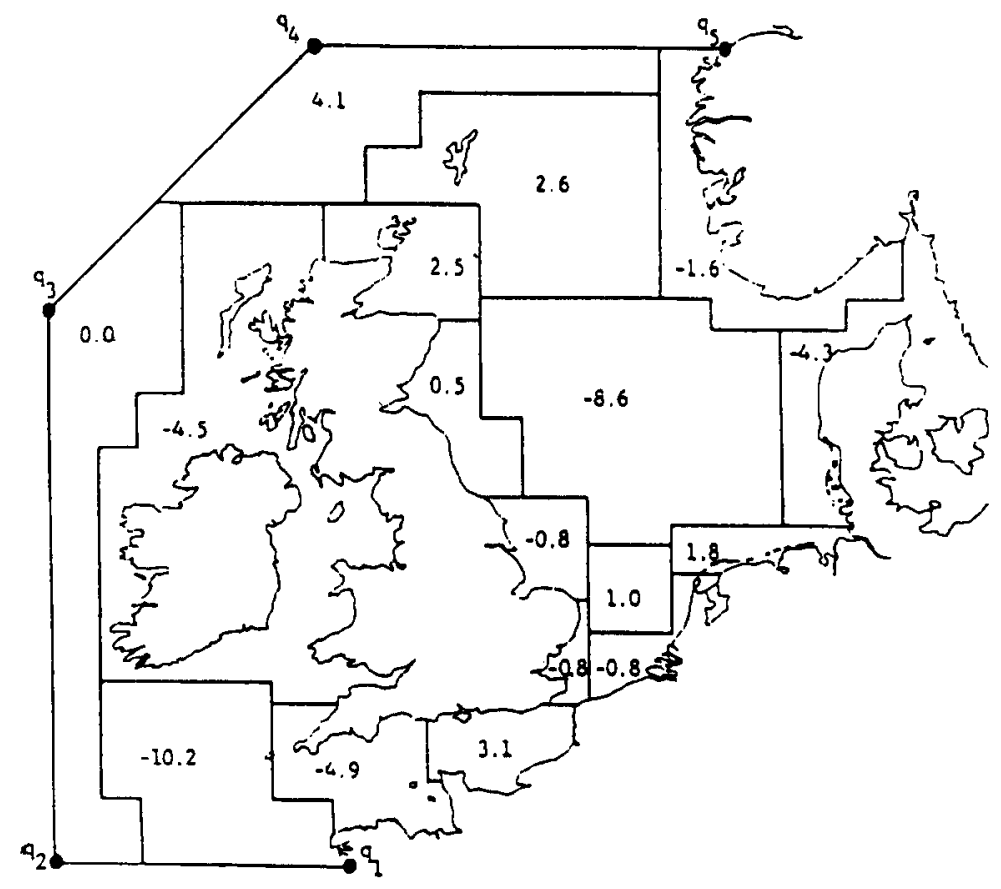

Figure 13. The adjustment of depth (m)

For CSM-16, with a prescribed water level as an open boundary condition, we introduce $q_{i}$, $i=1, \ldots, 5$, at the edges of the open boundary (see Figure 13). In between, the correction of the open boundary condition is found by linear interpolation.

In all the real life applications mentioned in this article, the operational model serves as our reference to evaluate the results. We have frequently compared the values of the criterion $\mathscr{T}$ or the RMS errors in the data observation points. Since the partition of $\boldsymbol{\Omega}$ into subdomains $\boldsymbol{\Omega}_{\boldsymbol{i}}^{\boldsymbol{C}}$ (to estimate $c_{i}$ ) and $\Omega_{j}^{D}$ (to estimate $d_{j}$ ) is the same as was used in Experiment II, the effect of estimating the uncertainty of the open boundary condition is illustrated by comparison with the results of Experiment II. By estimating only the depth and bottom friction parameters, the RMS errors were reduced by $16 \%$ in Experiment II. With the additional estimation of the uncertain open boundary condition in the present experiment, the reduction is $22 \%$ (see Table I). The RMS error in each data observation point is given in Column 3.

The adjustments of the depth in Experiment II are occasionally large (see Figure 6). If the boundary uncertainty is explicitly taken into account, the adjustments for the depth and bottom friction are much smaller (see Figure 13 and Table III), while the overall result (the reduction of the RMS errors) is improved. All the adjustments of the depth lie within a $2 \%$ bound. This is far below the acceptable level for the adjustments, i.e. between 5 and $10 \%$. Moreover, the smoothed boundary indicates that only a small additional force is needed (see Figure 14). The realizations of the processes $q_{2}$ and $q_{5}$ are very small, in an absolute sense they do not exceed $0.01 \mathrm{~m}$. The realizations of the other processes, $q_{1}, q_{3}$ and $q_{4}$, are somewhat larger, but they still do not exceed $0.035 \mathrm{~m}$. Furthermore, it is remarkable that $q_{1}, q_{2}$ and $q_{3}$ are strictly positive. We can conclude that the mean level of the prescribed open boundary must be increased. 


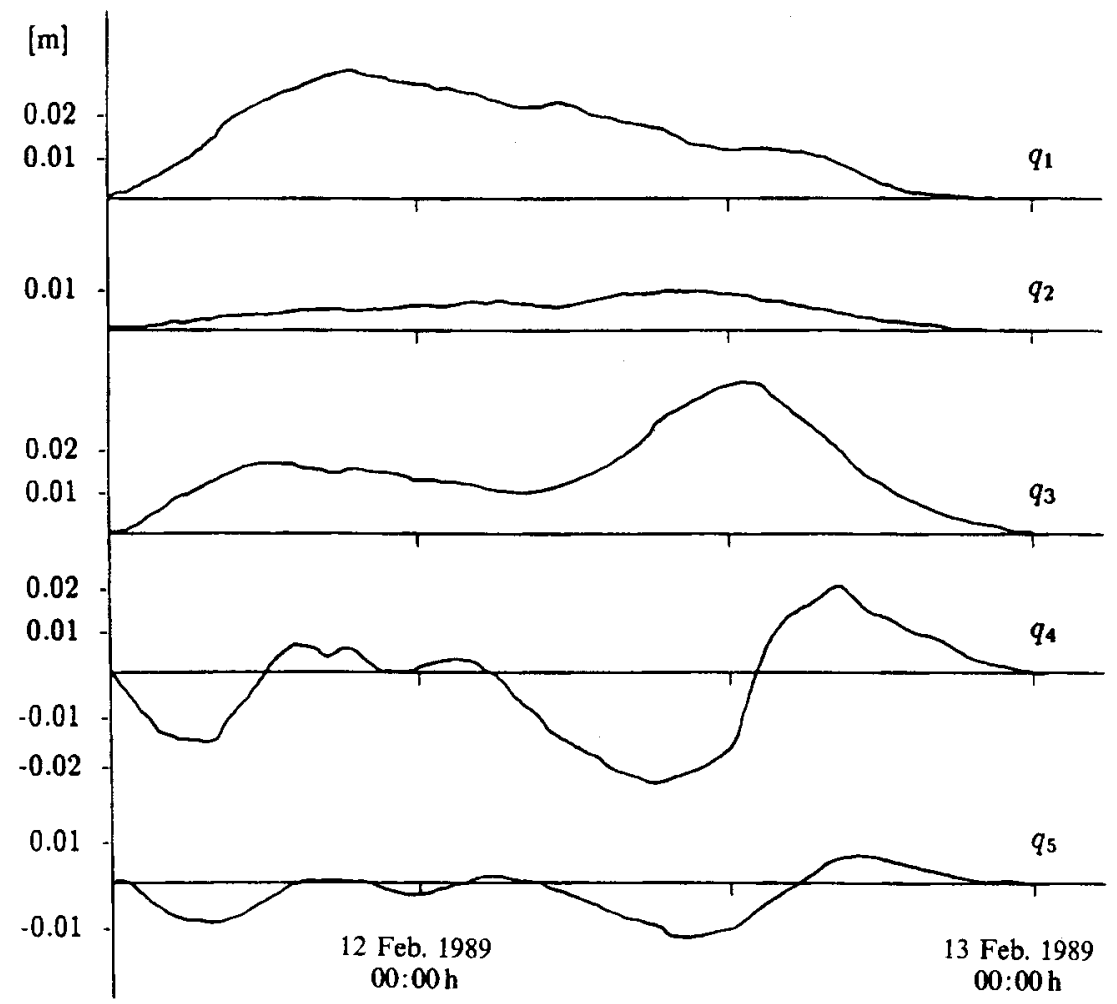

Figure 14. The smoothed correction at the edges of the open boundaries in CSM-16

Table III. The adjustments of the bottom friction coefficients (compared to those in the operational model)

\begin{tabular}{lrrrc}
\hline & $c_{1}$ & $c_{2}$ & $c_{3}$ & $c_{4}$ \\
\hline Experiment II & 17 & -10 & 8 & -3 \\
Experiment III & 8 & -10 & 2 & -1 \\
\hline
\end{tabular}

We have seen that the performance of the simulation model is improved by explicitly estimating the uncertain open boundaries, not only with respect to the RMS errors but also with respect to the physical interpretation of the adjustments of the coefficients. Without the estimation of the open boundary, the depth was partly corrected for the neglected open boundary errors. This implies that the depth is adjusted for very specific open boundary errors and it makes the adjustments dependent on the actual situation. Therefore, in this case the improved simulation performance does not necessarily imply that the prediction capability of the model is also increased. By comparison with the results of Experiment III (Figure 15) we conclude that the adjustments of the depth in Experiment II are in fact partly a compensation for uncertainties in the open boundary prescription. This compensational aspect makes the adjustments hard to interpret in a physical way and limits the prediction capability of the tidal model. 


\section{CONCLUSIONS}

In this paper a parameter estimation method is developed based on a gradient oriented minimization algorithm. One of the crucial aspects in deriving a suitable method was the work of Chavent. According to Chavent's method, the exact gradient of an error criterion can be computed by combining the solutions of the original model simulation and its adjoint model.
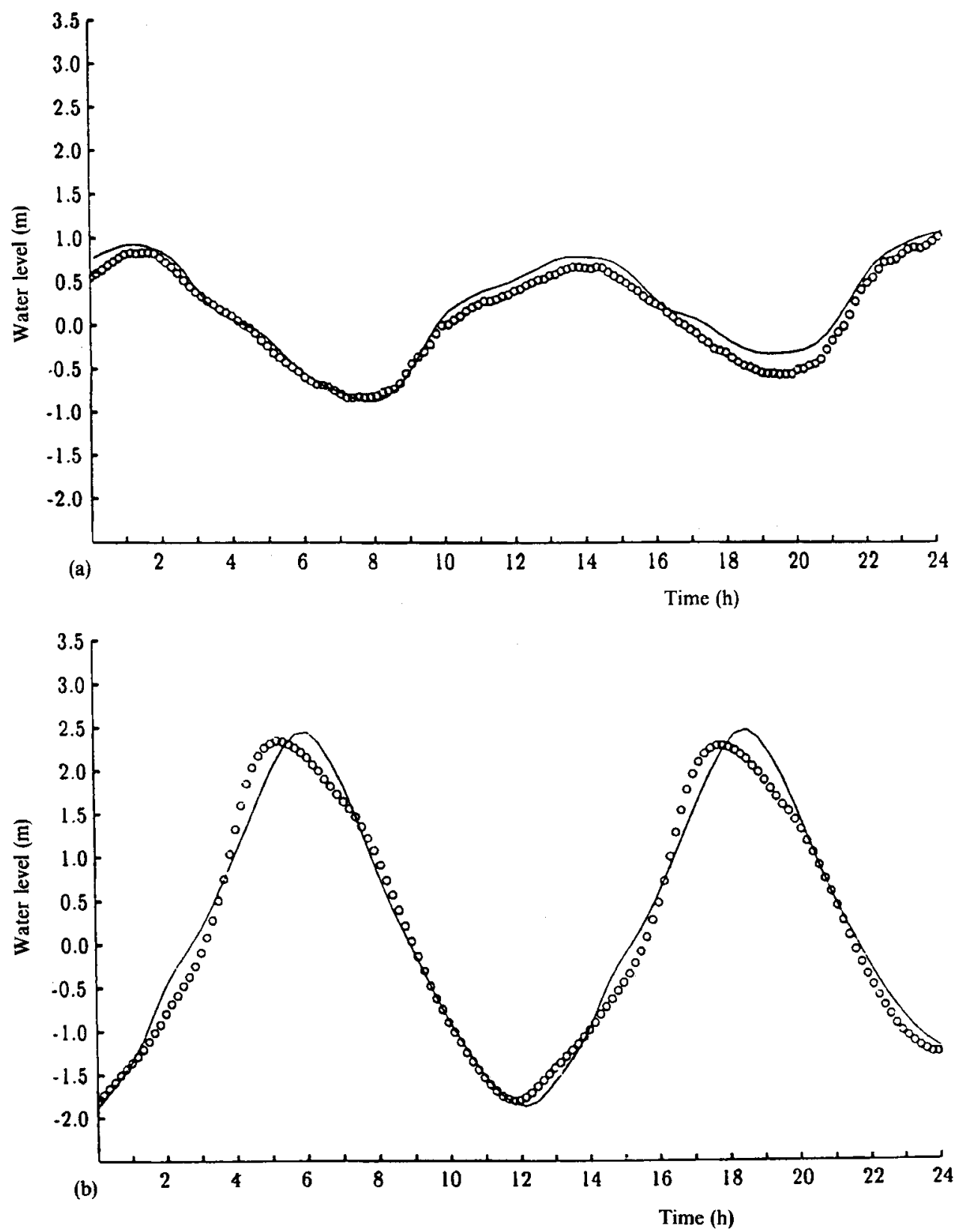

Figure 15. The effects of estimating the uncertainties in the open boundary conditions on 12 February 1989.000 , field data; -, CSM-16, Experiment III. (a) Lowestoft; (b) Vlissingen; (c) IJmuiden 


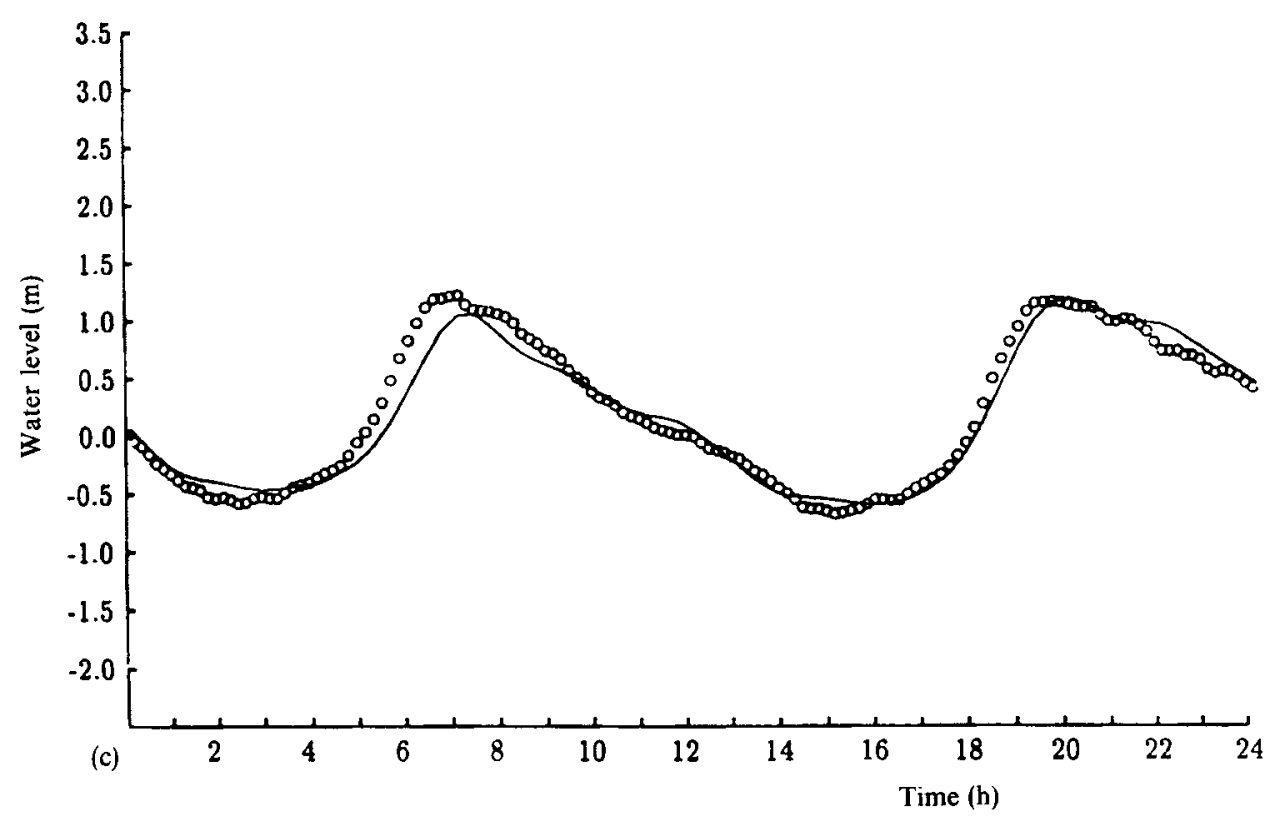

Figure 15. (Continued)

This algorithm for parameter estimation has several advantages.

(a) It is flexible, in the sense that the number and type of unknown parameters can easily be changed. The adjoint model is invariant and only the way of combining the simulation results with the adjoint solution is different.

(b) It is computationally efficient, since the computational time for each iteration step is independent of the number of unknown parameters.

(c) It is applicable to large scale models.

In order to be able to estimate parameters in models with uncertain boundary conditions, a state estimation has to be included. The proposed strategy considers the equations which are satisfied by the state estimates as constraints for the minimization of the error criterion with respect to the parameters. For the estimation of the state, a Kalman smoother is employed. The state estimations can now be conceived as deterministic state variables. This implies that the method of Chavent can again be used to determine the gradient of the error criterion.

The primary aim of estimating parameters is to improve the performance of the simulation model, which is expressed by the RMS errors in the data observation points. From all our experiments, we conclude that these RMS errors are reduced substantially. By minimizing the error criterion, the adjustments of the model parameters account for all the model errors. This implies that these parameter adjustments partly compensate for other model errors. This effect can be reduced by introducing stochastic open boundary conditions.

\section{REFERENCES}

1. P. G. J. ten Brummelhuis, B. de Jong and A. W. Heemink, 'Stochastic dynamic approach to predict water levels in estuaries', J. Hydraul. Eng., 114, 1339-1358 (1988).

2. P. G. J. ten Brummelhuis and A. W. Heemink, 'Parameter identification in tidal models with uncertain boundary conditions', Stochastic Hydrol. Hydraul., 4, 193-208 (1990). 
3. H. Cox, 'On the estimation of state variables for noisy dynamical systems', IEEE Trans. Automatic Control, AC-9, 5-12 (1964).

4. D. M. Detchmendy and R. Sridhar, 'Sequential estimation of states and parameters in noisy non-linear dynamical systems', Proc. Joint Automatic Control Conf., Troy, NY, 1965.

5. R. E. Mortensen, 'Maximum likelihood recursive nonlinear filtering', J. Optimization theory Appl., 2, 386-394 (1968).

6. G. Chavent, 'Identification of distributed parameter systems: about the least-squares method, its implementation and identifiability', Proc. 5th IF AC Symp. Identification and System Parameter Estimation, 1979.

7. J. J. Dronkers, Tidal Computations in Rivers and Coastal Waters, North-Holland, Amsterdam, 1964.

8. G. S. Stelling, 'On the construction of computational methods for shallow water flow problems', Rijkswaterstaat Communications, 1983.

9. R. Fletcher, Practical Methods of Optimization, Vols. I and 2, Wiley, Chichester, 1981.

10. H. T. Banks and K. Kunisch, Estimation Techniques for Distributed Parameter Systems, Birkhauser, Boston, MA, (1989).

11. P. G. J. ten Brummelhuis, 'Parameter estimation in tidal models with uncertain boundary conditions', Ph.D. Thesis, University of Twente, 1992.

12. A. W. Heemink, Two-dimensional shallow water flow identification, Appl. Math. Modeling, 12, 109-118 (1988).

13. A. H. Jazwinski, Stochastic Processes and Filtering Theory, Academic Press, New York, 1970.

14. M. Morf, S. S. Sidhu and T. Kailath, 'Some new algorithms for recursive estimation in constant, linear, discrete time systems', IEEE Trans. Automatic Control, AC-19, (1974).

15. G. K. Verboom, J. G. De Ronde and R. P. van Dijk, 'A fine grid tidal flow and storm surge model of the North Sea', Continental Shelf Res., 1992.

16. G. L. Geernaert, 'Bulk parameterizations for the wind stress and heat fluxes', in G. L. Geernaert and W. L. Plant (eds), Surface Waves and Fluxes, I. Current Theory, Kluwer, Dordrecht, 1990, pp. 91-172.

17. S. A. Kitaigorodskii, The Physics of Air-Sea Interaction, Israel Program for Scientific Translations, Jerusalem, 1973 (translated from Russian).

18. G. L. Geernaert, K. B. Katsaros and K. Richter, 'Variation of the drag coefficient and its dependence on sea state', J. Geophys. Res., 91, 7667-7679 (1986).

19. A. E. Bryson and Y. C. Ho, Applied Optimal Control, Ginn, Waltham, 1969.

20. Chao-Lin Chiu (Ed.), Applications of Kalman Filter Theory to Hydrology, Hydraulics and Water Resources, University of Pittsburg, PA, 1978.

21. J. J. Leendertse, 'A water quality simulation model for well mixed estuaries and coastal seas, Part 1: Principles of computation', Rand Corporation Memorandum RM-6230, Santa Monica, CA, 1970. 